Journal of Mathematical Logic

(C) World Scientific Publishing Company

\& Singapore University Press

\title{
Linear orders: when embeddability and epimorphism agree
}

\author{
Riccardo Camerlo \\ Dipartimento di matematica, Università di Genova, Via Dodecaneso 35, 16146 Genova - Italy; \\ camerlo@dima.unige.it \\ Raphaël Carroy \\ Kurt Gödel Research Center, Währinger Strasse 25, 1090 Wien - Austria; \\ raphael.carroy@univie.ac.at \\ Alberto Marcone \\ Dipartimento di Scienze Matematiche, Informatiche e Fisiche, Università di Udine, Via delle \\ Scienze 208,33100 Udine —Italy; alberto.marcone@uniud.it \\ Received (Day Month Year) \\ Revised (Day Month Year)
}

\begin{abstract}
When a linear order has an order preserving surjection onto each of its suborders we say that it is strongly surjective. We prove that the set of countable strongly surjective linear orders is a $\check{\mathrm{D}}_{2}\left(\boldsymbol{\Pi}_{1}^{1}\right)$-complete set. Using hypotheses beyond ZFC, we prove the existence of uncountable strongly surjective orders.

Keywords: Linear orders, epimorphisms, effective descriptive set theory, strongly surjective linear orders

Mathematics Subject Classification 2010: 03E15, 06A05, 03E65
\end{abstract}

\section{Introduction}

There are two natural ways of comparing a pair of linear orders $L$ and $M$ : embeddability and epimorphism. We write $L \leq_{i} M$ when there is an order preserving injection, also called an embedding, from $L$ to $M$. Similarly, $L \leq_{s} M$ stands for the existence of an order preserving surjection, also called an epimorphism, from $M$ onto $L$. The equivalence relation associated to $\leq_{s}$ is written $\equiv_{s}$.

Using the axiom of choice, $L \leq_{s} M$ implies $L \leq_{i} M$, but the embeddability relation $\leq_{i}$ is in general weaker than the relation $\leq_{s}$ induced by epimorphisms. For example, the ordinal number $\omega$ embeds into $\omega+1$, but there is no epimorphism from $\omega+1$ onto $\omega$.

There are however linear orders $M$ for which the relations $L \leq_{i} M$ and $L \leq_{s} M$ turn out to be equivalent. The ordinals satisfying this property have been characterized in [4] (see Theorem 1.2 below). The aim of this article is to study the class 
of orders $M$ for which the two notions coincide.

For the purpose of this paper, when talking about a linear order, we will always assume that it is non-empty; in particular, if no contrary mention is given, when a linear order is written as a sum $\sum_{i \in I} L_{i}$, all the summands are assumed to be non-empty.

We are now ready to give our main definition.

Definition 1.1. A linear order $M$ is strongly surjective if, for any linear order $L$, $L \leq_{i} M$ implies $L \leq_{s} M$; equivalently, if $M$ surjects order-preservingly onto each of its suborders.

The following characterization of strongly surjective ordinals is Corollary 29 of [4].

Theorem 1.2. An ordinal is strongly surjective if and only if it is a finite multiple of an indecomposable countable ordinal, that is, if it is of the form $\omega^{\alpha} m$, for some $\alpha<\omega_{1}$ and $m>0$.

The rationals are also strongly surjective: indeed by Proposition 16(1) in 4] $L \leq_{s} \mathbb{Q}$ for every countable linear order $L$. Up to $\equiv_{s}, \mathbb{Q}$ is the only countable non-scattered strongly surjective order (recall that $L$ is scattered if $\mathbb{Q} \leq_{i} L$ ): see Proposition 2.2 below.

Our main result is the following classification of the descriptive complexity of the set of countable strongly surjective linear orders:

Theorem 1.3. The set of countable strongly surjective orders is $\check{\mathrm{D}}_{2}\left(\boldsymbol{\Pi}_{1}^{1}\right)$-complete.

Here $\check{\mathrm{D}}_{2}\left(\boldsymbol{\Pi}_{1}^{1}\right)$ is the class of sets which are union of an analytic and a coanalytic set. The set we are interested in belongs to this class because the set of scattered strongly surjective orders is $\boldsymbol{\Pi}_{1}^{1}$, while the set of non-scattered strongly surjective orders is $\boldsymbol{\Sigma}_{1}^{1}$. In fact they are both complete in their respective classes (Corollary 4.19 and Proposition 3.3).

Our proof of the upper bound for scattered strongly surjective orders makes an essential use of both effective descriptive set theory and the fact that $\leq_{s}$ is a well quasi-order on the countable linear orders. The latter is the main theorem of $[9]$ and [4.

Even if the study of the first two levels of the projective hierarchy is a longstanding topic, examples of sets that are true $\boldsymbol{\Delta}_{2}^{1}$ (that is, $\boldsymbol{\Delta}_{2}^{1}$ but neither analytic nor coanalytic) are very rare. The interest in these sets has recently been rekindled by Fournier's study of the difference hierarchy of co-analytic sets ([6]). However, as far as we know, the set of countable strongly surjective orders is the first concrete example of a $\check{\mathrm{D}}_{2}\left(\boldsymbol{\Pi}_{1}^{1}\right)$-complete set that is not made so by design. Furthermore, two natural examples of sets which are complete in the dual class $\mathrm{D}_{2}\left(\boldsymbol{\Pi}_{1}^{1}\right)$ (consisting of the intersections of an analytic and a coanalytic set) were found in [5] and [1].

Here is the plan of the paper. 
In Section 2 we prove some basic properties of strongly surjective linear orders, and we present a useful way of defining epimorphisms by pieces, that we use throughout the paper.

We start studying the descriptive complexity of the set of countable strongly surjective linear orders in Section 3 The set Lin of all linear orders $\leq_{K}$ on a subset $K$ of $\mathbb{N}$ is Polish as it is a closed subspace of $2^{\mathbb{N} \times \mathbb{N}}$. We then call StS the set of strongly surjective orders in Lin. Definition 1.1 immediately gives an upper bound, StS being indeed a $\Pi_{2}^{1}$ subset of Lin. We prove in this section that StS is $\check{\mathrm{D}}_{2}\left(\boldsymbol{\Pi}_{1}^{1}\right)$ hard (Theorem 3.11). Our proof uses a study of the powers of $\mathbb{Z}$ and we notably prove that $\mathbb{Z}^{K}$ is strongly surjective for all countable $K$.

In Section 4 we show that for any countable scattered linear order $K$, there is a $\Delta_{1}^{1}(K)$ function that maps a linear order $L$ to an epimorphism from $K$ to $L$ when it exists, and to the refusing symbol $\perp$ otherwise (Theorem 4.17). As a corollary we get a $\check{D}_{2}\left(\boldsymbol{\Pi}_{1}^{1}\right)$ definition of StS (Corollary 4.20). This completes the proof of Theorem 1.3 .

Finally, Section 5 deals with uncountable linear orders. We first prove that many concrete (e.g. $\mathbb{R}, \mathbb{R} \backslash \mathbb{Q}$, their finite products, and also $\mathbb{R}^{\mathbb{N}}, \mathbb{Q}^{\mathbb{N}}, 2^{\alpha}$ for $\alpha<\omega_{1}$ ) are not strongly surjective, leaving open the problem of the provability in ZFC of the existence of an uncountable strongly surjective linear order. By contrast, we prove the existence of uncountable strongly surjective orders assuming either PFA (Theorem 5.13) or the existence of what we call a Baumgartner tree (Theorem 5.18). The latter hypothesis is connected to the principle $\diamond^{+}$, and thus orthogonal to PFA.

We conclude by discussing some problems that remain open and suggest new lines of research.

\subsection{About notations}

Variable symbols $K, L, M$ always stand for linear orders. $L^{\star}$ stands for the reverse of the linear order $L$. We call equimorphism the equivalence relation $\equiv_{i}$ associated to $\leq_{i}$, and we use the symbol $\simeq$ to denote isomorphism. The notation for operations such as sums and products on linear orders is standard; a reference is [15. In particular, $\sum_{i \in I} L_{i}$ is the sum ordered by $I$ of disjoint copies of each $L_{i}$, in other words $\bigcup_{i \in I}\{i\} \times L_{i}$ ordered lexicographically. The multiplicative notation $L K$ stands for $K$ copies of $L$, i.e. $\sum_{k \in K} L$.

Given an order $\left(K, \leq_{K}\right)$, and $p \in K$, define $(\leftarrow, p]_{K}$ as $\left\{n \in K \mid n \leq_{K} p\right\}$ and order it with the order induced by $\leq_{K}$. Define in a similar fashion the orders $(\leftarrow, p)_{K},(p, q)_{K},(p, q]_{K},[p, q)_{K},[p, q]_{K},(p, \rightarrow)_{K}$ and $[p, \rightarrow)_{K}$. We allow the notation $[p, q]_{K}$ when $p=q$ as well, letting then $[p, p]_{K}=\{p\}$. All these sets (including $\left.(\leftarrow, \rightarrow)_{K}=K\right)$ will be called intervals.

A subset $K^{\prime}$ of $K$ is convex when $x, y \in K^{\prime}$ and $x \leq_{K} y$ imply $[x, y]_{K} \subseteq K^{\prime}$ (so every interval is convex, but not all convex sets are intervals).

We call $\operatorname{Emb}(L, K)$ the set of all embeddings from $L$ to $K$, and $\operatorname{Epi}(L, K)$ the set of all epimorphisms from $K$ onto $L$. 


\section{Strong surjectivity}

We begin by stating some basic properties of strongly surjective orders.

\section{Proposition 2.1.}

1. A linear order $L$ is strongly surjective if and only if $L^{\star}$ is.

2. If $L$ is strongly surjective and $M \leq_{i} L \leq_{s} M$, then $M$ is strongly surjective and $L \equiv{ }_{s} M$.

3. If $L$ and $M$ are strongly surjective and $L \equiv_{i} M$, then $L \equiv_{s} M$.

Proof. (1) is obvious. (2) Let $K \leq_{i} M$. Since $M \leq_{i} L$ and $L$ is strongly surjective, there is an epimorphism $L \rightarrow K$. As there is also an epimorphism $M \rightarrow L$, this yields $K \leq_{s} M$. (3) follows from the definition of strongly surjective.

Part (3) of Proposition 2.1 states that in any class of equimorphism there is at most one $\equiv_{s}$-class of strongly surjective orders. However, not every class of equimorphism contains a strongly surjective order. Indeed, for an ordinal number $\alpha$ the classes of equimorphism, isomorphism and bi-epimorphism coincide. So if $\alpha$ is not of the form given by Theorem 1.2. its equimorphism class does not contain any strongly surjective order.

The results of 4] easily yield the following characterizations of countable strongly surjective linear orders that are not scattered:

Proposition 2.2. Let $L$ be a countable non-scattered linear order. The following are equivalent:

1. $L$ is strongly surjective;

2. $\mathbb{Q} \leq_{s} L$;

3. L has no initial or final segment which is scattered.

Proof. The equivalence of (1) and (2) follows because all countable non-scattered linear orders are equimorphic and $\mathbb{Q}$ is strongly surjective. By the above observation $L$ is strongly surjective if and only if $L \equiv_{s} \mathbb{Q}$, which in turn is equivalent to $\mathbb{Q} \leq_{s} L$ because $L \leq_{s} \mathbb{Q}$ for every countable $L$ by Proposition 16(1) in [4].

The equivalence of (2) and (3) follows from Proposition 17 in [4].

Definition 2.3. Given a linear order $L$ without a maximum, the cofinality of $L$, denoted $\operatorname{cof}(L)$, is the smallest ordinal number $\alpha$ such that there exists an increasing function $\alpha \rightarrow L$ unbounded above in $L$.

Similarly, for a linear order $L$ without a minimum, the coinitiality of $L$, denoted $\operatorname{coi}(L)$, is the reverse $\alpha^{\star}$ of the smallest ordinal $\alpha$ such that there exists an increasing function $\alpha^{\star} \rightarrow L$ unbounded below in $L$. Equivalently, $\operatorname{coi}(L)=\left(\operatorname{cof}\left(L^{\star}\right)\right)^{\star}$.

Recall that $L$ is short means that $\omega_{1} \not_{i} L$ and $\omega_{1}{ }^{\star} \not_{i} L$. 


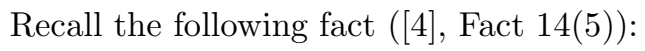

Proposition 2.4. If $K$ and $L$ have no maximum and $K \leq_{s} L$, then $\operatorname{cof}(K)=$ $\operatorname{cof}(L)$. Similarly, if $K, L$ have no minimum and $K \leq_{s} L$, then $\operatorname{coi}(K)=\operatorname{coi}(L)$.

\section{Proposition 2.5.}

1. If a strongly surjective order has a minimum, then it is a well-order. If it has a maximum, then it is the reverse of a well-order.

2. A strongly surjective linear order that is not an ordinal has coinitiality $\omega^{\star}$. Similarly, a strongly surjective linear order that is not the reverse of an ordinal has cofinality $\omega$.

3. Every strongly surjective linear order is short.

4. The cardinality of a strongly surjective linear order cannot exceed the continuum

Proof. (1) If $L$ is a strongly surjective order with a minimum and $K$ is a non-empty subset of $L$, then $K$ must have a minimum, otherwise $K \leq_{s} L$ would be impossible. Similarly for the maximum.

(2) If $L$ is an ill-founded strongly surjective order, then $\omega^{\star} \leq_{i} L$ and so $\omega^{\star} \leq_{s} L$. It also follows that $L$ does not have a minimum. So $\operatorname{coi}(L)=\omega^{\star}$ by Proposition 2.4 . Similarly for the cofinality.

(3) By Proposition 2.4. (1) and (2) any suborder of a strongly surjective order $L$ must have either a maximum or cofinality $\omega$. Therefore $\omega_{1} \not_{i} L$. Similarly $\omega_{1}{ }^{\star} \mathbb{Z}_{i} L$.

(4) follows from (3) and a classical theorem of Urysohn's ([15, Theorem 9.28) about short linear orders.

It is useful to give a name to the orders satisfying the necessary conditions for strong surjectivity given in the first two items of Proposition 2.5.

Definition 2.6. A linear order $L$ is admissible if the following conditions hold:

1. $L$ has a miminum or it has coinitiality $\omega^{\star}$;

2. $L$ has a maximum or it has cofinality $\omega$.

So an order is short if and only if it and all of its suborders are admissible.

\subsection{Defining epimorphisms}

Given non-empty convex subsets $K_{0}$ and $K_{1}$ of $K$, say that $K_{0} \leq K_{1}$ when for all $x \in K_{0}$ and $y \in K_{1}$ we have $x \leq_{K} y$; similarly define $K_{0}<K_{1}$ if for all $x \in K_{0}$ and all $y \in K_{1}$ one has $x<_{K} y$. Say that $K_{0}$ and $K_{1}$ are adjacent if $K_{0} \leq K_{1}$ and there is no $x \in K$ satisfying $K_{0}<\{x\}<K_{1}$. Say they are connected when $K_{0} \leq K_{1}$ but $K_{0} \nless K_{1}$ (so that they share an element).

An epimorphism can be defined on a covering by convex sets.

Definition 2.7. We say that a family of non-empty convex sets $\left(K_{i}\right)_{i \in I}$ of an order 
$K$ is nice if and only if the index set $I$ is an interval of $\mathbb{Z}$, the family $\left(K_{i}\right)_{i \in I}$ is unbounded above and below in $K$ and for all $i \in I, K_{i} \leq K_{i+1}$ holds.

We say that $\left(K_{i}\right)_{i \in I}$ is a nice covering of $K$ if it is a covering of $K$ by a nice family.

Lemma 2.8 (Definition by pieces). Suppose we have $\left(K_{i}\right)_{i \in I}$ a nice family of convex subsets of $K$ and $\left(L_{i}\right)_{i \in I}$ a nice covering of $L$ satisfying that for any $i \in I$ when $K_{i}$ and $K_{i+1}$ are not adjacent then $L_{i}$ has a maximum or $L_{i+1}$ has a minimum, and if $K_{i}$ and $K_{i+1}$ are connected, so are $L_{i}$ and $L_{i+1}$.

If for all $i \in I, L_{i} \leq_{s} K_{i}$ holds, then $L \leq_{s} K$.

Proof. Take $\sigma_{i} \in \operatorname{Epi}\left(L_{i}, K_{i}\right)$ and whenever $K_{i}$ and $K_{i+1}$ are not adjacent let $l_{i}$ be the maximum of $L_{i}$ if it exists, or the minimum of $L_{i+1}$ otherwise. Define then the map $\sigma: K \rightarrow L$ as follows.

$$
\sigma(x)= \begin{cases}\sigma_{i}(x) & \text { if } x \in K_{i} \text { for some } i \in I \\ l_{i} & \text { if } K_{i}<\{x\}<K_{i+1} \text { for some } i \in I\end{cases}
$$

We defined $\sigma$ on every $K_{i}$ and on the convex sets between $K_{i}$ and $K_{i+1}$, so on all of $K$. Let us first check that it is well-defined. Suppose $x$ is in $K_{i} \cap K_{i+1}$ for some $i \in I$. Then, since $K_{i} \leq K_{i+1}$, we have $K_{i} \cap K_{i+1}=\{x\}$, so that $x=\max K_{i}=\min K_{i+1}$ connects the two intervals. The hypothesis gives that $\max L_{i}=\min L_{i+1}$, and as the maps $\sigma_{i}$ and $\sigma_{i+1}$ are epimorphisms we have

$$
\sigma_{i}(x)=\sigma_{i}\left(\max K_{i}\right)=\max L_{i}=\min L_{i+1}=\sigma_{i+1}\left(\min K_{i+1}\right)=\sigma_{i+1}(x),
$$

so $\sigma$ is indeed well-defined. Since the maps $\sigma_{i}$ are epimorphisms and the sets $L_{i}$ form a nice covering of $L$, we finally have $\sigma \in \operatorname{Epi}(L, K)$.

In the above proof, we say that $\sigma$ is defined by pieces. Some specific operations come in handy to define epimorphisms by pieces.

Definition 2.9. Given $K, L$ linear orders, $\sigma \in \operatorname{Epi}(L, K)$ and $l \in L$, we denote $\sigma^{l}$ the following epimorphism:

$$
\begin{aligned}
\sigma^{l}: K & \longrightarrow(\leftarrow, l]_{L} \\
k & \longmapsto \begin{cases}\sigma(k) & \text { if } \sigma(k) \leq_{L} l \\
l & \text { otherwise }\end{cases}
\end{aligned}
$$

Similarly we define

$$
\begin{aligned}
\sigma_{l}: K & \longrightarrow[l, \rightarrow)_{L} \\
k & \longmapsto \begin{cases}\sigma(k) & \text { if } \sigma(k) \geq_{L} l \\
l & \text { otherwise. }\end{cases}
\end{aligned}
$$


Given $l \leq_{L} l^{\prime}$, we also define

$$
\begin{gathered}
\sigma_{l}^{l^{\prime}}: K \longrightarrow\left[l, l^{\prime}\right]_{L} \\
k \longmapsto \begin{cases}l & \text { if } \sigma(k)<l \\
\sigma(k) & \text { if } l \leq \sigma(k) \leq l^{\prime} \\
l^{\prime} & \text { if } l^{\prime}<\sigma(k) .\end{cases}
\end{gathered}
$$

Proposition 2.10 (Family mash). Given linear orders $K$ and $L$, with $L$ admissible, if there is a nice family $\left(K_{i}\right)_{i \in I}$ of $K$ such that $L \leq_{s} K_{i}$ holds for all $i \in I$, then we have $L \leq_{s} K$.

Proof. We may assume that $L$ is not a singleton, and so no $K_{i}$ is a singleton. Moreover, we can suppose that $I$ has more than one element, otherwise if $I=\{i\}$ then $K_{i}=K$ (because $K_{i}$ is convex and unbounded) and we are done.

For each $i \in I$ fix $\sigma_{i} \in \operatorname{Epi}\left(L, K_{i}\right)$. We want to define $\sigma \in \operatorname{Epi}(L, K)$ by pieces.

Suppose first that $I$ is finite, and let $i$ and $j$ be its minimum and maximum. Notice that $\left(K_{i}, K_{j}\right)$ is nice. On the $L$ side, take any $l \in L$ and consider the nice connected covering $\left((\leftarrow, l]_{L},[l, \rightarrow)_{L}\right)$. Then we can use Lemma 2.8, with $\left(\sigma_{i}\right)^{l}$ and $\left(\sigma_{j}\right)_{l}$ witnessing $(\leftarrow, l]_{L} \leq_{s} K_{i}$ and $[l, \rightarrow)_{L} \leq_{s} K_{j}$ respectively, to define $\sigma$.

From now on, we suppose that $I$ is infinite. There are four cases.

1. When $L$ has a minimum $l_{0}$ and a maximum $l_{1}$, choose $i \in I$ different from the minimum and maximum of $I$ (at most one of the extrema exists), and let $K^{-}=\left\{k \in K \mid\{k\}<K_{i}\right\}$ and $K^{+}=\left\{k \in K \mid K_{i}<\{k\}\right\}$. The nice covering $\left(K^{-}, K_{i}, K^{+}\right)$of $K$ and the connected covering $\left(\left\{l_{0}\right\}, L,\left\{l_{1}\right\}\right)$ of $L$ allow the definition by pieces of $\sigma$.

2. When $L$ has a minimum $\hat{l}$ and no maximum, we need to distinguish two subcases.

If $I$ has a maximum $i$ we consider the nice covering $\left(K \backslash K_{i}, K_{i}\right)$ and the connected covering $(\{\hat{l}\}, L)$.

If instead $I$ has no maximum, by admissibility of $L$ let $\left\{l_{n}\right\}_{n \in \mathbb{N}}$ be strictly increasing and cofinal in $L$. Fix $i \in I$ and let $K_{i}^{\prime}=K_{i} \cup\left\{k \in K \mid\{k\}<K_{i}\right\}$. Now consider the nice family $\left(K_{i}^{\prime}, K_{i+1}, K_{i+2}, \ldots\right)$ and the nice connected covering

$$
\left(\left[\hat{l}, l_{i}\right]_{L},\left[l_{i}, l_{i+1}\right]_{L},\left[l_{i+1}, l_{i+2}\right]_{L}, \ldots\right) .
$$

Using $\left(\sigma_{i}\right)^{l_{i}}$ and $\left(\sigma_{j}\right)_{l_{j-1}}^{l_{j}}$ for $j>i$ we again get the definition by pieces of $\sigma$.

3. When $L$ has a maximum and no minimum, we can just mirror the previous case.

4. When $L$ has no extrema, we look for a connected nice covering $\left(L_{i}\right)_{i \in I}$ to match $\left(K_{i}\right)_{i \in I}$. Take $\left(l_{i}\right)_{i \in \mathbb{Z}}$ strictly increasing, coinitial and cofinal in $L$. If $I=\mathbb{Z}$ then take $\left(\left[l_{i}, l_{i+1}\right]_{L}\right)_{i \in I}$. If $I$ has a minimum $j$, take $L_{j}=\left(\leftarrow, l_{j}\right]_{L}$ and $\left(\left[l_{i}, l_{i+1}\right]_{L}\right)_{i \geq j}$. If $I$ has a maximum $j$, take $\left(\left[l_{i}, l_{i+1}\right]_{L}\right)_{i<j}$ and $L_{j}=\left[l_{j}, \rightarrow\right)_{L}$. In any case, we can use the appropriate $\left(\sigma_{i}\right)_{l_{i}}^{l_{i+1}},\left(\sigma_{j}\right)_{l_{j}}$, and $\left(\sigma_{j}\right)^{l_{j}}$ to define $\sigma$ by pieces.

In the above proof we say that we mash the family $\left(K_{i}\right)_{i \in I}$ onto $L$.

Corollary 2.11. For $K$ and $L$ admissible, we have $L \leq_{s} L K$. 
Proof. By admissibility of $K$, take $\left(k_{i}\right)_{i \in I}$ increasing, coinitial and cofinal in $K$ for some $I$ which is an interval in $\mathbb{Z}$. Mash the nice family $\left(L \times\left\{k_{i}\right\}\right)_{i \in I}$ of $L K$ onto $L$ using Proposition 2.10

\subsection{Operations on strongly surjective orders}

In general, the sum of strongly surjective orders is not strongly surjective: consider for example a countable ordinal whose Cantor normal form has two summands and use Theorem 1.2. We now show instead that the product of two strongly surjective orders is still strongly surjective, and that the left-quotient of a strongly surjective order by a scattered order is also strongly surjective. First note the following.

Proposition 2.12. Let $I$ be any order and for each $i \in I$, let $L_{i}$ be a strongly surjective order. Then $L=\sum_{i \in I} L_{i}$ is strongly surjective if and only if for every non-empty $J \subseteq I$ there is an epimorphism from $L$ onto $L_{J}=\sum_{j \in J} L_{j}$.

Proof. If $L$ is strongly surjective, then it must admit an epimorphism onto its suborder $L_{J}$ for any non-empty $J \subseteq I$.

Conversely, suppose there is an epimorphism $\psi_{J}: L \rightarrow L_{J}$ for any non-empty $J \subseteq I$. Let $K$ be a suborder of $L$ and let $J$ be the set of indices $j$ such that $K$ intersects $L_{j}$ in a non-empty set $K_{j}$, so that $K=\sum_{j \in J} K_{j}$. Since each $L_{j}$ is strongly surjective, let $\varphi_{j}: L_{j} \rightarrow K_{j}$ be an epimorphism. These induce an epimorphism $\varphi: L_{J} \rightarrow K$. Then $\varphi \circ \psi_{J}: L \rightarrow K$ is an epimorphism.

This yields the following simple examples of strongly surjective orders.

Example 2.13. Let $\gamma, \delta$ be countable ordinals and $n, m>0$. Then $\left(\omega^{\gamma} n\right)^{\star}, \omega^{\delta} m$ and $\left(\omega^{\gamma} n\right)^{\star}+\omega^{\delta} m$ are strongly surjective.

Proof. The fact that $\left(\omega^{\gamma} n\right)^{\star}, \omega^{\delta} m$ are strongly surjective is a consequence of Theorem 1.2 and Proposition 2.1(1). So by Proposition 2.12 it is enough to show $\left(\omega^{\gamma} n\right)^{\star} \leq_{s}\left(\omega^{\gamma} n\right)^{\star}+\omega^{\delta} m$ and $\omega^{\delta} m \leq_{s}\left(\omega^{\gamma} n\right)^{\star}+\omega^{\delta} m$, which can be done by a definition by pieces using the presence of an extremum in the range.

Corollary 2.14. If $L$ and $M$ are strongly surjective, then $L M$ is strongly surjective. In particular $L^{n}$ is strongly surjective for all $n \in \mathbb{N}$.

Proof. By Proposition 2.12, it is enough to show $L K \leq_{s} L M$ for any suborder $K$ of $M$. Let $\varphi: M \rightarrow K$ be an epimorphism and for $k \in K$ let $M_{k}=\varphi^{-1}(\{k\})$. As $M$ is strongly surjective, each $M_{k}$ must be admissible. Since $L$ is also admissible by Proposition 2.5. Corollary 2.11 implies that there is an epimorphism $\varphi_{k}: L M_{k} \rightarrow L$ for every $k \in K$. Gluing together these epimorphisms yields $L K \leq_{s} L M$.

Strongly surjective orders are not closed under infinite products (ordered lexicographically), as we will show in Section 5 .

Lemma 2.15. If $L$ is a scattered linear order and $0<n<m$, then $L m \not_{i} L n$. 
Proof. We first show the special case $L 2 \not_{i} L$, which is actually Lemma 1.17 of [13. Notice that if $L 2 \leq_{i} L$ then an easy induction shows that $L n \leq_{i} L$ for any $n$. We show that under this hypothesis $L$ is not scattered. To this end we recursively define for every $s \in 2^{<\mathbb{N}}$ a subset $L_{s}$ of $L$ which is isomorphic to $L$ and a point $x_{s} \in L$. Start with $L_{\emptyset}=L$. Assuming that $L_{s} \simeq L$ and $L_{s} \subseteq L$, since $L 3 \leq_{i} L_{s}$, pick a point $x_{s}$ in the middle copy of $L$ embedded in $L_{s}$ and let $L_{s 0}$ and $L_{s 1}$ be the left and right copies of $L$ embedded in $L_{s}$. Then $\left\{x_{s}\right\}_{s \in 2<\mathbb{N}}$ is a dense suborder of $L$, and so $L$ is not scattered.

Now assume $0<n<m$ and $L m \leq_{i} L n$. Again inductively one can show that $L(m+k(m-n)) \leq_{i} L n$ for all $k$. If $k$ is large enough we have $m+k(m-n) \geq 2 n$ and hence $\operatorname{Ln} 2 \leq_{i} L n$, which by the above implies that $L n$ is not scattered. Thus $L$ is not scattered.

Proposition 2.16. If $L$ is scattered and $L K$ is strongly surjective, then $K$ is strongly surjective.

Proof. Let $J \leq_{i} K$ and fix an epimorphism $\varphi: L K \rightarrow L J$. Define the relation $R \subseteq K \times J$ by letting $k R j \Leftrightarrow \varphi(L \times\{k\}) \cap(L \times\{j\}) \neq \emptyset$. If $k \in K$ we denote by $R_{k}$ the vertical section $\{j \in J \mid k R j\}$. Similarly, for $j \in J, R^{j}$ is the horizontal section $\{k \in K \mid k R j\}$. Notice that:

- all sections are non-empty (i.e. the domain of $R$ is $K$ and its range is $J$ );

- all sections are convex subsets of the respective linear order;

- $\left|R_{k}\right| \leq 3$ for each $k \in K$ (by Lemma 2.15).

To define an epimorphism $\psi: K \rightarrow J$, we only need to define a surjection $\psi$ that satisfies $k R \psi(k)$ for all $k \in K$. Given $k \in K$ we distinguish several cases.

(a) If $\left|R_{k}\right|=1$ and $j$ is the unique element of $R_{k}$ set $\psi(k)=j$.

(b) If there is $j$ (necessarily unique, by Lemma2.15) such that $L \times\{j\} \subseteq \varphi(L \times\{k\})$, then set $\psi(k)=j$; note that if $\left|R_{k}\right|=3$ then $k$ satisfies this case.

(c) So it remains to define $\psi$ on the set $H$ of those $k$ such that $\left|R_{k}\right|=2$, but do not fall in case (b). Consider $I$ a maximal $\leq_{K}$-convex subset of $H$ that is embeddable

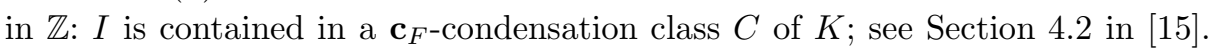
So $I$ has order type finite, $\omega, \omega^{\star}$ or $\omega^{\star}+\omega$. We need to define $\psi$ on each such $I$.

(c1) Suppose first that $I$ has $n$ elements $k_{0}<\ldots<k_{n-1}$. Consequently, $\bigcup_{r=0}^{n-1} R_{k_{r}}$ consists of $n+1$ consecutive points of $J$, say $j_{0}<\ldots<j_{n}$, so that $R_{k_{r}}=$ $\left\{j_{r}, j_{r+1}\right\}$.

(c1a) If $k_{0}=\min C$, set $\psi\left(k_{r}\right)=j_{r+1}$ for $0 \leq r \leq n-1$. Notice that in this case, since $j_{0}$ does not witness that case (b) applies to $k_{0}, R^{j_{0}}$ consists of $k_{0}$ and an infinite convex set with supremum $k_{0}$ : then for all but at most one $k \in R^{j_{0}} \backslash\left\{k_{0}\right\}$ we have $\psi(k)=j_{0}$ by case (a).

(c1b) If $k_{0} \neq \min C$ but $k_{n-1}=\max C$, then let $\psi\left(k_{r}\right)=j_{r}$ for $0 \leq r \leq n-1$. An argument similar to the one used in case (c1a) shows that in this case $\psi(k)=j_{n}$ for some $k$. 
(c1c) If neither $k_{0}$ is the first element of $C$, nor $k_{n-1}$ is the last element of $C$, let $k^{\prime}$ be the immediate precedessor of $k_{0}$ and $k^{\prime \prime}$ be the immediate successor of $k_{n-1}$ in $K$. Thus $k^{\prime} R j_{0}, k^{\prime \prime} R j_{n}$ (because neither $k_{0}$ nor $k_{n-1}$ satisfy the condition of case (b)) and both $\psi\left(k^{\prime}\right)$ and $\psi\left(k^{\prime \prime}\right)$ have been defined according to cases (a) or (b). Notice that $\psi\left(k^{\prime}\right)$ and $\psi\left(k^{\prime \prime}\right)$ cannot have both been defined according to clause (b), with values different from $j_{0}, j_{n}$, respectively, as in this case one would have $L(n+3) \leq_{i} L(n+2)$, contradicting Lemma 2.15. This implies that either $\psi\left(k^{\prime}\right)=j_{0}$ or $\psi\left(k^{\prime \prime}\right)=j_{n}$. If $\psi\left(k^{\prime}\right)=j_{0}$, let $\psi\left(k_{r}\right)=j_{r+1}$; otherwise, let $\psi\left(k_{r}\right)=j_{r}$.

(c2) If $I$ has order type $\omega, \omega^{\star}$ or $\omega^{\star}+\omega$, then $\bigcup_{k \in I} R_{k}$ has the same order type and we can define $\psi$ on $I$ as any order preserving surjection onto $\bigcup_{k \in I} R_{k}$.

By construction, $\psi: K \rightarrow J$ is order preserving and surjective.

\section{Bounding the complexity of StS from below}

The closure properties of Subsection 2.2 allow to build several examples of strongly surjective linear orders. We present here other kinds of examples allowing to obtain some hardness results.

First we make our formal setting precise. We call Lin the subset of $2^{\mathbb{N} \times \mathbb{N}}$ consisting of all linear orders $\leq_{K}$ on a subset $K=\operatorname{dom}\left(\leq_{K}\right)$ of $\mathbb{N}$. By definition it is a Polish subspace of $2^{\mathbb{N} \times \mathbb{N}}$. To avoid heavy notations, when there is no possible confusion we just write $K$ for the pair $\left(K, \leq_{K}\right)$.

When we work with elements of Lin we fix recursive copies of $\mathbb{N}$ and $\mathbb{Q}$, denoted respectively by $\omega$ and $\eta$. Moreover we assume a fixed way of implementing sums (finite or infinite) and products as recursive (and hence continuous) operations which produce new elements of Lin.

Remark 3.1. In the literature most often people work with LO, the space of all total orders on the domain $\mathbb{N}$. The downside of LO is the absence of finite orders, which we need for the main result in Section 4. That is why we deal with Lin. However, for the classification results on strongly surjective orders the two settings are equivalent. Indeed, denote by Fin the $\Sigma_{2}^{0}$ set of finite orders in Lin, and notice that there are continuous functions LO $\rightarrow$ Lin $\backslash$ Fin and Lin $\backslash$ Fin $\rightarrow$ LO that preserve order types. If $\Gamma$ is a pointclass that includes $\boldsymbol{\Sigma}_{2}^{0}$ and is closed under finite unions and continuous preimages, and the set of strongly surjective orders in LO belongs to $\Gamma$, then StS $\backslash$ Fin $\in \Gamma$, so StS $\in \Gamma$. Conversely, if StS $\in \Gamma$, one has that the strongly surjective orders as a subset of LO are in $\Gamma$ as well.

\subsection{Basic hardness}

Let Scat and WO be the subsets of Lin consisting of the scattered countable linear orders and of the countable well-orders. It is well-known that both Scat and WO 
are $\Pi_{1}^{1}$ and $\Pi_{1}^{1}$-complete.

Proposition 3.2. The sets $\mathrm{StS}, \mathrm{StS} \cap \mathrm{Scat}$ and $\mathrm{StS} \cap \mathrm{WO}$ are $\Pi_{1}^{1}$-hard.

Proof. Let $g:$ Lin $\rightarrow$ Lin be defined by $g(L)=(1+L) \omega$. Using Proposition 2.5 and Theorem 1.2 as $g(L)$ has a minimal element for any $L$, we have $g(L) \in$ StS if and only if $g(L) \in$ WO. Since $g(L) \in$ WO if and only if $L \in$ WO, we have that $g$ reduces WO to StS, to StS $\cap$ Scat, and to StS $\cap$ WO as well.

We now consider the set of countable strongly surjective linear orders that are non-scattered.

Proposition 3.3. The set $\mathrm{StS}$ is $\boldsymbol{\Sigma}_{1}^{1}$-hard, and the set of non-scattered strongly surjective orders is $\Sigma_{1}^{1}$ and $\boldsymbol{\Sigma}_{1}^{1}$-complete.

Proof. Let $f:$ Lin $\rightarrow$ Lin be defined by $f(L)=\eta+L \omega$. As $f(L)$ is non-scattered for all $L$, we have $f(L) \in$ StS if and only if it has no scattered initial nor final segments by Proposition 2.2. But $f(L)$ never has a scattered initial segment, and it has a scattered final segment if and only if $L$ itself is scattered. So finally $f$ reduces Lin $\backslash$ Scat to StS, and even to the set of non-scattered strongly surjective countable linear orders, which are consequently $\Sigma_{1}^{1}$-hard.

The fact that StS $\backslash$ Scat is $\Sigma_{1}^{1}$ follows from the characterization of Proposition 2.2.(ii).

\subsection{Powers of $\mathbb{Z}$}

The main new ingredient needed for the lower bound is a general version of the exponentiation with base $\mathbb{Z}$. There are two definitions of $\mathbb{Z}^{\alpha}$ for $\alpha$ an ordinal number. The first one is by ordinal induction (see [15], Definition 5.34), while the second ([15], Definition 5.35) is a direct set theoretic definition, and it can actually be used as a definition of $\mathbb{Z}^{K}$ for $K$ any linear order. As pointed out in [15], Exercise 5.36(1), the two definitions coincide when $K$ is a well-order.

We first recall the definition by ordinal induction.

\section{Definition 3.4.}

1. $\mathbb{Z}^{0}=1$,

2. $\mathbb{Z}^{\alpha+1}=\mathbb{Z}^{\alpha} \omega^{\star}+\mathbb{Z}^{\alpha}+\mathbb{Z}^{\alpha} \omega$,

3. $\mathbb{Z}^{\alpha}=\left(\sum_{\beta<\alpha} \mathbb{Z}^{\beta} \omega\right)^{\star}+1+\sum_{\beta<\alpha} \mathbb{Z}^{\beta} \omega$ if $\alpha$ is a limit ordinal.

The following equalities will be useful.

Proposition 3.5. For any $\alpha$ and $\beta<\alpha$, we have

$$
\mathbb{Z}^{\alpha}=\left(\sum_{\gamma<\alpha} \mathbb{Z}^{\gamma} \omega\right)^{\star}+1+\sum_{\gamma<\alpha} \mathbb{Z}^{\gamma} \omega=\left(\sum_{\beta \leq \gamma<\alpha} \mathbb{Z}^{\gamma} \omega\right)^{\star}+\sum_{\beta \leq \gamma<\alpha} \mathbb{Z}^{\gamma} \omega .
$$


Proof. To prove the first equality we argue by induction on $\alpha$. The cases $\alpha=0$ and $\alpha$ limit are immediate from the definition. For the successor case we have

$$
\begin{aligned}
\mathbb{Z}^{\alpha+1} & =\mathbb{Z}^{\alpha} \omega^{\star}+\mathbb{Z}^{\alpha}+\mathbb{Z}^{\alpha} \omega \\
& =\mathbb{Z}^{\alpha} \omega^{\star}+\left(\sum_{\gamma<\alpha} \mathbb{Z}^{\gamma} \omega\right)^{\star}+1+\sum_{\gamma<\alpha} \mathbb{Z}^{\gamma} \omega+\mathbb{Z}^{\alpha} \omega \\
& =\left(\sum_{\gamma<\alpha+1} \mathbb{Z}^{\gamma} \omega\right)^{\star}+1+\sum_{\gamma<\alpha+1} \mathbb{Z}^{\gamma} \omega,
\end{aligned}
$$

where in the central step we use the induction hypothesis.

The second equality can be proved applying the first one to $\beta$, using $\left(\mathbb{Z}^{\beta} \omega\right)^{\star}+$ $\mathbb{Z}^{\beta} \omega=\mathbb{Z}^{\beta} \mathbb{Z}=\mathbb{Z}^{\beta+1}$.

Proposition 3.6. For any countable ordinal $\alpha$ and natural number $m>0$, the order $\mathbb{Z}^{\alpha} m$ is strongly surjective.

Proof. Since finite linear orders are trivially strongly surjective, by Corollary 2.14 it suffices to show that each $\mathbb{Z}^{\alpha}$ is strongly surjective. Proceed by induction on $\alpha$. When $\alpha=0$ we get the singleton linear order. Notice that $\mathbb{Z}$ is strongly surjective by Example 2.13, so that Corollary 2.14 handles the successor step because $\mathbb{Z}^{\alpha+1}=$ $\mathbb{Z}^{\alpha} \mathbb{Z}$

Suppose now that $\delta$ is limit and that $\mathbb{Z}^{\gamma}$ is strongly surjective for all $\gamma<\delta$. By Corollary 2.14] so are $\mathbb{Z}^{\gamma} \omega$ and $\mathbb{Z}^{\gamma} \omega^{\star}$. Recall that, by Proposition 3.5, $\mathbb{Z}^{\delta}$ can be written as a sum over the index set $I=\delta^{\star}+\delta$ : $\mathbb{Z}^{\delta}=\left(\sum_{\gamma<\delta} \mathbb{Z}^{\gamma} \omega\right)^{\star}+\sum_{\gamma<\delta} \mathbb{Z}^{\gamma} \omega$.

First we show that if $1 \leq \beta_{0}<\beta_{1} \leq \delta$ and $H$ is a non-empty subset of $\delta$ with $\sup H<\beta_{0}$, then

$$
\sum_{\gamma \in H} \mathbb{Z}^{\gamma} \omega \leq_{s} \sum_{\beta_{0} \leq \gamma<\beta_{1}} \mathbb{Z}^{\gamma} \omega
$$

If $\beta_{1}=\rho+1$ is a successor ordinal, $\left(\mathbb{Z}^{\beta_{0}} \omega, \mathbb{Z}^{\rho} \omega\right)$ is a nice family in $\sum_{\beta_{0} \leq \gamma<\beta_{1}} \mathbb{Z}^{\gamma} \omega$ and we can mash $\sum_{\beta_{0} \leq \gamma<\beta_{1}} \mathbb{Z}^{\gamma} \omega$ onto $\sum_{\gamma \in H} \mathbb{Z}^{\gamma} \omega$, since $\sum_{\gamma \in H} \mathbb{Z}^{\gamma} \omega \leq_{i} \mathbb{Z}^{\beta_{0}} \leq_{i}$ $\mathbb{Z}^{\beta_{0}} \omega \leq_{i} \mathbb{Z}^{\rho} \omega$. If $\beta_{1}$ is limit, let $\rho_{n}$ be an increasing cofinal sequence in $\beta_{1}$, with $\rho_{0}=\beta_{0}$ : we can mash the nice family $\left(\mathbb{Z}^{\rho_{n}} \omega\right)_{n \in \mathbb{N}}$ onto $\sum_{\gamma \in H} \mathbb{Z}^{\gamma} \omega$. So, in either case we get (3.1).

Take now a non-empty subset $J$ of $I$ : it determines two subsets $J^{-}, J^{+} \subseteq \delta-$ one of them possibly empty - and a suborder $K=\left(\sum_{\gamma \in J^{-}} \mathbb{Z}^{\gamma} \omega\right)^{\star}+\sum_{\gamma \in J^{+}} \mathbb{Z}^{\gamma} \omega$. We want to show that $K \leq_{s} \mathbb{Z}^{\delta}$, so that we can conclude the proof by applying Proposition 2.12. Set $K^{-}=\left(\sum_{\gamma \in J^{-}} \mathbb{Z}^{\gamma} \omega\right)^{\star}$ and $K^{+}=\sum_{\gamma \in J^{+}} \mathbb{Z}^{\gamma} \omega$.

First notice that we may suppose that both $K^{-}$and $K^{+}$are non-empty. In fact if, for example, $K^{-}=\emptyset$, letting $\alpha=\min J^{+}$, we have

$$
K=K^{+}=\mathbb{Z}^{\alpha}+K^{+} \leq_{s}\left(\mathbb{Z}^{\alpha} \omega\right)^{\star}+K^{+},
$$

so that an epimorphism from $\mathbb{Z}^{\delta}$ onto the rightmost part gives by composition an epimorphism onto $K$. 
Similarly, we may assume that both $J^{-}$and $J^{+}$are unbounded in $\delta$. Indeed, if $\alpha<\delta$ is an upper bound for, say, $J^{+}$, we have

$$
K \leq_{s} K^{-}+\mathbb{Z}^{\alpha+1} \leq_{s} K^{-}+\mathbb{Z}^{\alpha+1} \omega \leq_{s} K^{-}+\sum_{\alpha+2 \leq \gamma<\delta} \mathbb{Z}^{\gamma} \omega,
$$

where in the first inequality we used the induction hypothesis, and in the last one (3.1).

Since $\mathbb{Z}^{\delta}=\left(\sum_{\beta<\gamma<\delta} \mathbb{Z}^{\gamma} \omega\right)^{\star}+\sum_{\beta \leq \gamma<\delta} \mathbb{Z}^{\gamma} \omega$ for any $\beta<\delta$, it is enough to show that both $K^{-} \leq_{s}\left(\sum_{\beta \leq \gamma<\delta} \mathbb{Z}^{\gamma} \omega\right)^{\star}$ and $K^{+} \leq_{s} \sum_{\beta \leq \gamma<\delta} \mathbb{Z}^{\gamma} \omega$ hold for some $\beta$. To prove, for instance, the latter, let $\left\{\alpha_{n}\right\}_{n \in \mathbb{N}}$ be increasing and cofinal in $J^{+}$, with $\alpha_{0}=\min J^{+}$, and let $\left\{\beta_{n}\right\}_{n \in \mathbb{N}}$ be increasing and cofinal in $\delta$, with $\alpha_{n+1}<\beta_{n}$ for all $n \in \mathbb{N}$. Then, by (3.1), there exist epimorphisms

$$
\sum_{\beta_{n} \leq \gamma<\beta_{n+1}} \mathbb{Z}^{\gamma} \omega \rightarrow \sum_{\gamma \in J^{+}, \alpha_{n} \leq \gamma<\alpha_{n+1}} \mathbb{Z}^{\gamma} \omega
$$

Gluing them together, one obtains an epimorphism from $\sum_{\beta_{0} \leq \gamma<\delta} \mathbb{Z}^{\gamma} \omega$ onto $K^{+} \cdot \square$

Here is the set-theoretic definition of exponentiation with base $\mathbb{Z}$.

Definition 3.7. Let $K$ be a linear order. For any map $\varphi: K \rightarrow \mathbb{Z}$, $\operatorname{Supp}(\varphi)$ stands for the support of $\varphi$, that is $\operatorname{Supp}(\varphi)=\{k \in K \mid \varphi(k) \neq 0\}$. The $K$-power of $\mathbb{Z}$, denoted by $\mathbb{Z}^{K}$, is the following order on $\{\varphi: K \rightarrow \mathbb{Z} \mid \operatorname{Supp}(\varphi)$ is finite $\}$. If $\varphi, \psi: K \rightarrow \mathbb{Z}$ are maps with finite support let $\varphi \leq_{\mathbb{Z}^{K}} \psi$ if and only if $\varphi=\psi$ or $\varphi\left(k_{0}\right)<_{\mathbb{Z}} \psi\left(k_{0}\right)$ where $k_{0}=\max \{k \in \operatorname{Supp}(\varphi) \cup \operatorname{Supp}(\psi) \mid \varphi(k) \neq \psi(k)\}$.

We now show that if $K$ is countable but not a well-order then $\mathbb{Z}^{K} \equiv_{s} \mathbb{Q}$, and hence $\mathbb{Z}^{K}$ is strongly surjective by Proposition 2.2.

Lemma 3.8. For any linear orders $K$ and $L$ we have:

1. $\mathbb{Z}^{K+L} \simeq \mathbb{Z}^{K} \mathbb{Z}^{L}$

2. if $K$ is countable and with no minimum then $\mathbb{Z}^{K} \simeq \mathbb{Q}$.

Proof. (1) The bijection $\mathbb{Z}^{K+L} \rightarrow \mathbb{Z}^{K} \mathbb{Z}^{L}, \varphi \mapsto\left(\left.\varphi\right|_{K},\left.\varphi\right|_{L}\right)$ is an isomorphism.

(2) Take $K$ countable with no minimum, and suppose $\varphi<_{\mathbb{Z}^{K}} \psi$ holds for some $\varphi$ and $\psi$ in $\mathbb{Z}^{K}$. As $K$ has no minimum pick $k_{0} \in K$ that is strictly below every element of $\operatorname{Supp}(\varphi) \cup \operatorname{Supp}(\psi)$. Define $\varphi^{-}, \theta$ and $\psi^{+}$, all in $\mathbb{Z}^{K}$, as follows.

$$
\begin{gathered}
\varphi^{-}(x)= \begin{cases}-1 & \text { if } x=k_{0} \\
\varphi(x) & \text { otherwise }\end{cases} \\
\theta(x)= \begin{cases}1 & \text { if } x=k_{0} \\
\varphi(x) & \text { otherwise }\end{cases} \\
\psi^{+}(x)= \begin{cases}1 & \text { if } x=k_{0} \\
\psi(x) & \text { otherwise }\end{cases}
\end{gathered}
$$


We have

$$
\varphi^{-}<_{\mathbb{Z}^{K}} \varphi<_{\mathbb{Z}^{K}} \theta<_{\mathbb{Z}^{K}} \psi<_{\mathbb{Z}^{K}} \psi^{+},
$$

so $\mathbb{Z}^{K}$ is dense, countable, without extrema, giving $\mathbb{Z}^{K} \simeq \mathbb{Q}$.

Proposition 3.9. If $K$ is countable and not a well order then there is a countable ordinal $\alpha$ such that

$$
\mathbb{Z}^{K} \simeq \mathbb{Z}^{\alpha} \mathbb{Q}
$$

Hence $\mathbb{Z}^{K} \equiv{ }_{s} \mathbb{Q}$.

Proof. To obtain $\mathbb{Z}^{K} \simeq \mathbb{Z}^{\alpha} \mathbb{Q}$ it suffices to use the previous lemma with the decomposition $K=\alpha+K^{\prime}$ for $\alpha$ ordinal and $K^{\prime}$ without a minimum. Since $\mathbb{Z}^{K}$ is written as a $\mathbb{Q}$ sum we have $\mathbb{Q} \leq_{s} \mathbb{Z}^{K}$, which yields $\mathbb{Z}^{K} \equiv_{s} \mathbb{Q}$ because $K$, and hence $\mathbb{Z}^{K}$, is countable.

\subsection{The lower bound}

We now prove that StS is hard for the class $\check{\mathrm{D}}_{2}\left(\boldsymbol{\Pi}_{1}^{1}\right)$ of all sets that are the union of an analytic and a coanalytic set.

As we did for sums and products, we want to realize exponentiation with base $\mathbb{Z}$ as an operation on Lin. Since in this case the details are less straightforward, we provide them:

Proposition 3.10. There is a continuous (even recursive) function Lin $\rightarrow$ Lin mapping any $K$ to an order $\left(\zeta^{K}, \leq_{\zeta^{K}}\right)$ isomorphic to $\mathbb{Z}^{K}$.

Proof. Fix $n \mapsto s_{n}$ and $n \mapsto\left((n)_{0},(n)_{1}\right)$ recursive enumerations of $\mathbb{N}<\mathbb{N}$ and $\mathbb{N}^{2}$ respectively, as well as a recursive order $\prec_{\zeta}$, whose domain is the whole $\mathbb{N}$, that is isomorphic to the strict part of $\leq_{\mathbb{Z}}$. For any $K \in \operatorname{Lin}$ we define $\left(\zeta^{K}, \leq_{\zeta^{K}}\right) \in \operatorname{Lin}$.

First, the domain $\zeta^{K}$ is the set of codes for pairs of sequences of the same length, the first with values in $K$, the second in $\mathbb{N} \backslash\{0\}$. This simulates the finite support. For convenience we require the sequences with values in $K$ to be $\leq_{K}$-decreasing. Writing $\operatorname{lh}(s)$ for the length of a sequence $s$ :

$$
\begin{aligned}
\zeta^{K}=\left\{n \in \mathbb{N} \mid \operatorname{lh}\left(s_{(n)_{0}}\right)=\operatorname{lh}\left(s_{(n)_{1}}\right)\right. & \wedge \forall i<\operatorname{lh}\left(s_{(n)_{0}}\right) s_{(n)_{1}}(i) \neq 0 \\
& \left.\wedge \forall i<j<\operatorname{lh}\left(s_{(n)_{0}}\right) s_{(n)_{0}}(j)<_{K} s_{(n)_{0}}(i)\right\} .
\end{aligned}
$$

We now compare two codes of pairs of sequences on the first value on which they differ. We have to be careful because if the value differs on the first sequence, it means that the two sequences do not have the same support. Also, one sequence could extend the other. Formally, given $n, m \in \zeta^{K}$ we have $n \leq_{\zeta^{K}} m$ if and only if

- $n=m$

or 
- for all $i<\min \left\{\operatorname{lh}\left(s_{(n)_{0}}\right), \operatorname{lh}\left(s_{(m)_{0}}\right)\right\}$ we have

$$
\forall \varepsilon \in\{0,1\}\left(s_{(n)_{\varepsilon}}(i)=s_{(m)_{\varepsilon}}(i)\right),
$$

and

$$
\begin{aligned}
\left(\operatorname{lh}\left(s_{(n)_{0}}\right)<\operatorname{lh}\left(s_{(m)_{0}}\right) \wedge 0 \prec_{\zeta}\right. & \left.s_{(m)_{1}}\left(\operatorname{lh}\left(s_{(n)_{0}}\right)\right)\right) \vee \\
& \left(\operatorname{lh}\left(s_{(m)_{0}}\right)<\operatorname{lh}\left(s_{(n)_{0}}\right) \wedge s_{(n)_{1}}\left(\operatorname{lh}\left(s_{(m)_{0}}\right)\right) \prec_{\zeta} 0\right) ;
\end{aligned}
$$

or

- there exists $i<\min \left\{\operatorname{lh}\left(s_{(n)_{0}}\right), \operatorname{lh}\left(s_{(m)_{0}}\right)\right\}$ satisfying

$$
\forall j<i \forall \varepsilon \in\{0,1\}\left(s_{(n)_{\varepsilon}}(j)=s_{(m)_{\varepsilon}}(j)\right),
$$

and

$$
\begin{aligned}
& \left(s_{(n)_{0}}(i)<_{K} s_{(m)_{0}}(i) \wedge 0 \prec_{\zeta} s_{(m)_{1}}(i)\right) \vee \\
& \left(s_{(m)_{0}}(i)<_{K} s_{(n)_{0}}(i) \wedge s_{(n)_{1}}(i) \prec_{\zeta} 0\right) \vee \\
& \quad\left(s_{(n)_{0}}(i)=s_{(m)_{0}}(i) \wedge s_{(n)_{1}}(i) \prec_{\zeta} s_{(m)_{1}}(i)\right) .
\end{aligned}
$$

This is, given $K$, a recursive encoding of an order $\zeta^{K}$ isomorphic to $\mathbb{Z}^{K}$.

Theorem 3.11. The set $\mathrm{StS}$ is hard for the class $\check{\mathrm{D}}_{2}\left(\boldsymbol{\Pi}_{1}^{1}\right)$.

Proof. First observe that the set

$$
A=\{(K, L) \in \operatorname{Lin} \times \operatorname{Lin} \mid K \notin \mathrm{WO} \vee L \in \mathrm{WO}\}
$$

is $\check{\mathrm{D}}_{2}\left(\boldsymbol{\Pi}_{1}^{1}\right)$-complete: if $B=B_{0} \cup B_{1}$, with $f_{0}$ reducing $B_{0}$ to Lin $\backslash W O$ and $f_{1}$ reducing $B_{1}$ to WO, then $\left(f_{0}, f_{1}\right)$ reduces $B$ to $A$.

We now prove that $\mathrm{StS}$ continuously reduces $A$. To this end we use the continuous map $f$ defined by

$$
f(K, L)=\zeta^{K}(1+L) \omega .
$$

If $K \notin$ WO then by Proposition $3.9 \mathbb{Q} \leq_{s} \zeta^{K}$. Then $f(K, L)$ has no initial or final segment which is scattered and by Proposition 2.2 we have $f(K, L) \in \mathrm{StS}$. If $K \in \mathrm{WO}$ then $\zeta^{K}$ is isomorphic to an ordinal power of $\zeta$, which is scattered and strongly surjective by Proposition 3.6. In that case, using both Proposition 2.16 and Corollary 2.14, $f(K, L) \in \mathrm{StS}$ if and only if $(1+L) \omega \in \mathrm{StS}$. Finally, since $(1+L) \omega$ has a minimum, Proposition 2.5(1) and Theorem 1.2 tell us that $(1+L) \omega \in$ StS if and only if $L \in \mathrm{WO}$, which concludes the proof.

\section{Bounding the complexity of StS from above}

Given a set $A \neq \emptyset$, the spaces $A^{\mathbb{N}}$ and $A^{\mathbb{N} \times \mathbb{N}}$ are endowed with the product topology of the discrete topology on $A$.

Given $K, L$ in $\operatorname{Lin}, \operatorname{Emb}(L, K)$ is a closed subspace of $K^{L}$, so it is closed in $(\mathbb{N} \cup\{*\})^{\mathbb{N}}$ as well, where $*$ is a new symbol (we map the elements of $\mathbb{N} \backslash L$ to $*$ ). Similarly, the space $\operatorname{Epi}(L, K)$ is $\Pi_{2}^{0}$ in $L^{K}$ and hence a $\Pi_{2}^{0}$ subspace of $(\mathbb{N} \cup\{*\})^{\mathbb{N}}$. Therefore both $\operatorname{Emb}(L, K)$ and $\operatorname{Epi}(L, K)$ are Polish spaces. 


\subsection{Some effective facts}

We assume some familiarity with basic recursion theory. For effective descriptive set theory, we refer the reader to Section $3 \mathrm{E}$ of [14] or to [1]. Notice that Lin is a Polish recursive space in the sense of Section 2.4.3 in [11.

We make a heavy use of Chapter 4 of [14. Let us recall the following well-known facts ([14, 4D.3 and [1], Section 5.1.5, respectively). We state them in relativized form, fixing a parameter $K$.

Fact 4.1. If $X$ and $Y$ are recursive spaces and $\mathrm{A} \subseteq X \times Y$ is $\Pi_{1}^{1}(K)$ then $\{x \in X \mid$ $\left.\exists y \in \Delta_{1}^{1}(x, K)(x, y) \in \mathrm{A}\right\}$ is $\Pi_{1}^{1}(K)$.

Fact 4.2. Let $X$ be a recursive space, $Y$ a $\Pi_{1}^{1}(K)$ subset of a recursive space, and $\mathrm{A} \subseteq X \times Y$ a $\Pi_{1}^{1}(K)$ set. Then there exists a $\Delta_{1}^{1}(K)$ function $f: \exists^{\Delta} \mathrm{A} \rightarrow Y$ which uniformizes $\mathrm{A}$ on $\exists^{\Delta} \mathrm{A}$, where

$$
x \in \exists^{\Delta} \mathrm{A} \Longleftrightarrow \exists y \in \Delta_{1}^{1}(x, K)((x, y) \in \mathrm{A}) .
$$

Some basic operations on linear orders are effective.

Fact 4.3. The operation $\star: L \mapsto L^{\star}$ is recursive.

We now spell out what we mean by saying that the definition by pieces of Lemma 2.8 is $\Delta_{1}^{1}$.

Fact 4.4. The following sets are $\Delta_{1}^{1}$ :

1. for $I$ an interval of $\mathbb{Z}$, the set of $\left(K,\left(K_{i}\right)_{i \in I}\right)$ such that $K \in \operatorname{Lin}$ and $\left(K_{i}\right)_{i}$ is a nice family of $K$,

2. the same with nice covering,

3. the set of triples $(K, L, M)$ such that $L$ and $M$ are adjacent convex subsets of $K$,

4. the set of triples $(K, L, M)$ such that $L$ and $M$ are connected convex subsets of $K$,

5. the set of pairs $(L, n)$ such that $n$ is the maximum of $L$.

\section{Notation 4.5.}

- For $I$ an interval of $\mathbb{Z}$, Pieces $_{I}$ stands for the set of all $(\bar{K}, \bar{L}):=$ $\left(\left(K,\left(K_{i}\right)_{i \in I}\right),\left(L,\left(L_{i}\right)_{i \in I}\right)\right)$ in $\left(\operatorname{Lin} \times \operatorname{Lin}^{I}\right)^{2}$ such that: $\left(K_{i}\right)_{i \in I}$ is a nice family of convex subsets of $K ;\left(L_{i}\right)_{i \in I}$ is a nice covering of $L$; for any $i \in I$ when $K_{i}$ and $K_{i+1}$ are not adjacent then $L_{i}$ has a maximum or $L_{i+1}$ has a minimum; and if $K_{i}$ and $K_{i+1}$ are connected, so are $L_{i}$ and $L_{i+1}$.

- Call Epi the space $(\mathbb{N} \cup\{*\})^{\mathbb{N}} \cup\{\perp\}$ where $\perp$ is not an element of $(\mathbb{N} \cup\{*\})^{\mathbb{N}}$; Epi is equipped with the smallest Polish topology extending that of $(\mathbb{N} \cup\{*\})^{\mathbb{N}}$ and making $\{\perp\}$ a clopen set. 
Fact 4.6. For any interval $I$ of $\mathbb{Z}$, Pieces $_{I}$ is $\Delta_{1}^{1}$ and so is the map

$\operatorname{DefPieces}_{I}:$ Pieces $_{I} \times \mathrm{Epi}^{I} \longrightarrow$ Epi

$$
\left((\bar{K}, \bar{L}),\left(\sigma_{i}\right)_{i \in I}\right) \longmapsto \begin{cases}\sigma & \text { if } \forall i \in I \sigma_{i} \in \operatorname{Epi}\left(L_{i}, K_{i}\right) \\ \perp & \text { otherwise }\end{cases}
$$

where $\sigma \in \operatorname{Epi}(L, K)$ is given by Lemma 2.8 .

The explicit dependence on $I$ will be omitted, and we shall write simply DefPieces to denote this function.

Fact 4.7. If $K \in$ Scat then any convex suborder of $K$ is $\Delta_{1}^{1}(K)$.

Proof. Fix $K \in$ Scat. First notice that $K$ has countably many convex subsets (see [15], Exercise 5.33.1)). Moreover the set of convex suborders of $K$ is a $\Pi_{1}^{0}(K)$, and hence $\Sigma_{1}^{1}(K)$, subset of $2^{\mathbb{N} \times \mathbb{N}}$. So an application of Harrison's Effective Perfect Set Theorem ([14], Theorem 4F.1) concludes the proof.

Definition 4.8. Given $A$ and $B$ subsets of Lin, denote by $A+B$ the set

$\left\{K \in \operatorname{Lin} \mid \exists K_{0}\left(K_{0}\right.\right.$ is an initial segment of $\left.\left.K \wedge K_{0} \in \mathrm{A} \wedge K \backslash K_{0} \in \mathrm{B}\right)\right\}$.

Define then $n \cdot \mathrm{A}$ for $n \geq 1$ by induction on $n$ by $1 \cdot \mathrm{A}=\mathrm{A}$ and $(n+1) \cdot \mathrm{A}=n \cdot \mathrm{A}+\mathrm{A}$, finally $\operatorname{FinSum}(\mathrm{A})$ stands for $\bigcup_{n \in \mathbb{N}}(n+1) \cdot \mathrm{A}$.

Fact 4.9. If $A$ and $B$ are two $\Pi_{1}^{1}(K)$ subsets of Scat, then so are $A+B$ and FinSum $(A)$.

Proof. The class $\Pi_{1}^{1}(K)$ is closed by effective countable unions, so it suffices to prove the statement for A + B. This comes from Facts 4.7 and 4.1

\subsection{Uniformizations for epimorphisms}

Definition 4.10. Given $K \in \operatorname{Lin}$ we say that $K$ admits a $\Delta_{1}^{1}$-uniformization if there exists a $\Delta_{1}^{1}(K)$ map $\Phi_{K}:$ Lin $\rightarrow$ Epi such that $\Phi_{K}(L) \in \operatorname{Epi}(L, K)$ when $L \leq_{s} K$ and $\Phi_{K}(L)=\perp$ when $L \leq_{s} K$.

Given $\mathrm{A} \subseteq$ Lin, if all $K \in \mathrm{A}$ admit a $\Delta_{1}^{1}$-uniformization we say that $\mathrm{A}$ has the $\Delta_{1}^{1}$-uniformization for epimorphisms.

Our goal is to show in Theorem 4.17 below that Scat has the $\Delta_{1}^{1}$-uniformization for epimorphisms.

Fact 4.11. If $L$ admits a $\Delta_{1}^{1}$-uniformization, so does $L^{\star}$.

Proof. Define $\Phi_{L^{\star}}(M)=\Phi_{L}\left(M^{\star}\right)$.

Notice that a $\Delta_{1}^{1}$-uniformization of some $K \in \operatorname{Lin}$ is a $\boldsymbol{\Delta}_{1}^{1}$ subset of $\mathcal{U}=\operatorname{Lin} \times$ Epi. Following Section 5.1.1 in [11, we call $\left(\mathbf{D}^{1}, \mathbf{W}^{1}\right)$ the coding of $\boldsymbol{\Delta}_{1}^{1}$-subsets of $\mathcal{U}$. Recall that we have 
- $\mathbf{D}^{1}$ is a $\Pi_{1}^{1}$ subset of $\mathbb{N}^{\mathbb{N}}, \mathbf{W}^{1}$ is a $\Delta_{1}^{1}$ subset of $\mathbf{D}^{1} \times \mathcal{U}$

- $\left\{\mathbf{W}_{\alpha}^{1} \mid \alpha \in \mathbf{D}^{1}\right\}$ is the set of $\boldsymbol{\Delta}_{1}^{1}$ subsets of $\mathcal{U}$ and for any $K,\left\{\mathbf{W}_{\alpha}^{1} \mid \alpha \in\right.$ $\mathbf{D}^{1}$ and $\alpha$ recursive in $\left.K\right\}$ is the set of $\Delta_{1}^{1}(K)$ subsets of $\mathcal{U}$.

Fact 4.12. There is a partial $\Delta_{1}^{1}$ map: $\operatorname{Lin} \rightarrow \mathbf{D}^{1}, K \mapsto \alpha_{K}$ such that for every $K \in$ Lin admitting a $\Delta_{1}^{1}$-uniformization, $\alpha_{K} \in \mathbf{D}^{1}$ is a code of a $\Delta_{1}^{1}$-uniformization $\Phi_{K}=\mathbf{W}_{\alpha_{K}}^{1}$.

Proof. The relation

$$
\alpha \text { is the code of a function that uniformizes } K
$$

is a $\Pi_{1}^{1}$ subset of $\mathbf{D}^{1} \times$ Lin by definition:

$\alpha$ codes a uniformization of $K \Longleftrightarrow \alpha$ codes a function $\Phi$ and

$$
\forall L \in \operatorname{Lin}\left(\left(L \not \mathbb{s}_{s} K \wedge \Phi(L)=\perp\right) \vee \Phi(L) \in \operatorname{Epi}(L, K)\right) .
$$

The result then follows using Fact 4.2

Proposition 4.13. If $\mathrm{A}, \mathrm{B} \subseteq$ Scat have the $\Delta_{1}^{1}$-uniformization for epimorphisms, then so does $\mathrm{A}+\mathrm{B}$.

In particular, FinSum(A) has the $\Delta_{1}^{1}$-uniformization for epimorphisms.

Proof. We fix $K \in \mathrm{A}+\mathrm{B}$ and $K_{0} \in \mathrm{A}, K_{1} \in \mathrm{B}$ such that $K_{0}$ is an initial segment of $K$ and $K_{1}=K \backslash K_{0}$. Since $\mathrm{A}$ and $\mathrm{B}$ have the $\Delta_{1}^{1}$-uniformization for epimorphisms, for $i=0,1$ there exists a $\Delta_{1}^{1}$-uniformization $\Phi_{K_{i}}$ for $K_{i}$. As $K_{i}$ is a convex subset of $K \in$ Scat, by Fact $4.7 \Delta_{1}^{1}\left(K_{i}\right)$ is included in $\Delta_{1}^{1}(K)$. Recalling that each $\Phi_{K_{i}}$ is a $\Delta_{1}^{1}\left(K_{i}\right)$ map, this implies that $\Phi_{K_{i}}$ is a $\Delta_{1}^{1}(K)$ map.

By Lemma 2.8 (and using the fact that $K_{0}$ and $K_{1}$ are adjacent but not connected), $L$ satisfies $L \leq_{s} K$ if and only if there is a nice covering $\left(L_{0}, L_{1}\right)$ of $L$ satisfying $L_{0} \leq_{s} K_{0}$ and $L_{1} \leq_{s} K_{1}$. Since $\Phi_{K_{i}}$ allows to check whether $L_{i} \leq_{s} K_{i}$ in a $\Delta_{1}^{1}(K)$ way and using Fact $4.4(2)$, the set $\mathrm{C}$ of triples $\left(L, L_{0}, L_{1}\right)$ satisfying the latter is $\Delta_{1}^{1}(K)$.

We now use the effective version of Lusin-Novikov's "small section" uniformization result (see 14], 4F.6: the statement there is not effective, but the hint proves the effective version) to obtain two $\Delta_{1}^{1}(K)$ functions $\Psi_{0}$ and $\Psi_{1}$ with domain $\left\{L \mid \exists L_{0}, L_{1}\left(L, L_{0}, L_{1}\right) \in \mathrm{C}\right\}$ such that $\left(L, \Psi_{0}(L), \Psi_{1}(L)\right) \in \mathrm{C}$ holds for any $L \leq_{s} K$.

We can now define $\Phi_{K}$ : Lin $\rightarrow$ Epi by setting $\Phi_{K}(L)$ to be the map defined by pieces from $\Phi_{K_{0}} \circ \Psi_{0}(L)$ and $\Phi_{K_{1}} \circ \Psi_{1}(L)$ when $\Psi_{0}(L)$ and $\Psi_{1}(L)$ are defined, and $\Phi_{K}(L)=\perp$ otherwise. By Fact 4.6 , $\Phi_{K}$ is a $\Delta_{1}^{1}(K)$ map and in fact a $\Delta_{1}^{1}$-uniformization of $K$.

We recall (some version of) Hausdorff's hierarchy of countable scattered linear orders.

- Call Scat $\subseteq_{0} \subseteq$ Lin the class of singleton orders, 
- an element of Lin is in Scat ${ }_{\alpha}$ when it is isomorphic to a finite sum, an $\omega$-sum or an $\omega^{\star}$-sum of elements of $\bigcup_{\beta<\alpha} \operatorname{Scat}_{\beta}$.

Hausdorff proved that Scat $=\bigcup_{\alpha<\omega_{1}}$ Scat $_{\alpha}$, so for $K \in$ Scat we define the Hausdorff rank of $K$ :

$$
\operatorname{rk}_{\mathrm{H}}(K)=\min \left\{\alpha<\omega_{1} \mid K \in \mathrm{Scat}_{\alpha}\right\} .
$$

We have Scat ${ }_{\alpha}=\left\{K \in \mathrm{Scat} \mid \operatorname{rk}_{\mathrm{H}}(K) \leq \alpha\right\}$ and we can set Scat ${ }_{<\alpha}=\bigcup_{\beta<\alpha}$ Scat $_{\beta}=$ $\left\{K \in \mathrm{Scat} \mid \mathrm{rk}_{\mathrm{H}}(K)<\alpha\right\}$.

Recall that if $L$ is a suborder of $K$, then $\operatorname{rk}_{\mathrm{H}}(L) \leq \operatorname{rk}_{\mathrm{H}}(K)$ holds, and that $\operatorname{rk}_{\mathrm{H}}$ is a $\Pi_{1}^{1}$-norm (see [14], Section 4B). In particular, Scat ${ }_{\alpha}$ and Scat S $_{<\alpha}$ are $\Delta_{1}^{1}(\alpha)$. Moreover, for any $K \in$ Scat, using Theorem 4D.1(iii) of [14] we have $\operatorname{rk}_{\mathrm{H}}(K) \in$ $\Delta_{1}^{1}(K)$.

To prove that if Scat ${ }_{\alpha}$ has the $\Delta_{1}^{1}$-uniformization for epimorphism so does Scat ${ }_{\alpha+1}$ we only need to handle the case of $\omega$-sums (as for the case of $\omega^{\star}$-sums we can use Fact 4.11, and the case of finite sums is handled by Proposition 4.13). We use the following notion, implicitly used in 9 .

Definition 4.14. We say that an order $K$ is stable if and only if for all $k \in K$ we have $K \equiv_{s}[k, \rightarrow)_{K}$.

Since it is always the case that $[k, \rightarrow)_{K} \leq_{s} K, K$ stable really means $K \leq_{s}$ $[k, \rightarrow)_{K}$ for all $k \in K$. Notice that in particular a stable $K$ has a minimum and that the only stable orders with a maximum are the singletons.

Recall from [9] and [4 that the class of countable linear orders is well-quasiordered (wqo) under epimorphisms. In particular, ( $\left.\operatorname{Lin}, \leq_{s}\right)$ is well-founded. We use the following observation, due to Landraitis 9], Lemma 2.2.

Lemma 4.15. Every $K \in \operatorname{Lin}$ has a stable final segment.

Proof. Fix $K \in \operatorname{Lin}$ and look for $a \in K$ such that $[a, \rightarrow)_{K}$ is stable. Fix a sequence $\left(a_{n}\right)_{n \in \mathbb{N}}$ monotone and cofinal in $K$. The sequence $\left(\left[a_{n}, \rightarrow\right)_{K}\right)_{n \in \mathbb{N}}$ is $\leq_{s}$-decreasing so there is $N \in \mathbb{N}$ such that $\left(\left[a_{n}, \rightarrow\right)_{K}\right)_{n \geq N}$ is $\equiv_{s}$-constant, for $\leq_{s}$ is well-founded on Lin. Choose then $a=a_{N}$. For any $k \geq_{K} a$ there is $n>N$ such that $k \leq_{K} a_{n}$ holds, and finally

$$
[a, \rightarrow)_{K} \leq_{s}\left[a_{n}, \rightarrow\right)_{K} \leq_{s}[k, \rightarrow)_{K},
$$

so $[a, \rightarrow)_{K}$ is a stable final segment of $K$.

We need the following characterization of stability (also essentially contained in [9], Lemma 2.2).

Fact 4.16. An admissible linear order $K$ is stable if and only if it has a minimum and for all $a_{0} \leq_{K} a_{1}$ and $a$ in $K$ there are $b_{0}, b_{1}$ in $K$ such that $a \leq_{K} b_{0} \leq_{K} b_{1}$ and $\left[a_{0}, a_{1}\right]_{K} \leq_{s}\left[b_{0}, b_{1}\right]_{K}$. 
Proof. It is immediate that if $K$ is stable then it has the desired property, so it is enough to show the converse. Given any $k \in K$, take $\left(a_{i}\right)_{i \in \mathbb{N}}$ a cofinal monotone sequence in $K$, with $a_{0}=\min K$ and $a_{1}=k$. Use the hypothesis to find a sequence $\left(b_{i}^{0}, b_{i}^{1}\right)_{i \in \mathbb{N}}$ such that we have

- $a_{i+1} \leq_{K} b_{i}^{0} \leq_{K} b_{i}^{1} \leq_{K} b_{i+1}^{0}$,

- $\left[a_{i}, a_{i+1}\right]_{K} \leq_{s}\left[b_{i}^{0}, b_{i}^{1}\right]_{K}$.

Notice that since $\left[b_{0}^{0}, b_{0}^{1}\right]_{K} \leq_{s}\left[k, b_{0}^{1}\right]_{K}$ we can assume that $b_{0}^{0}=k$.

We can apply Lemma 2.8 to define by pieces a surjection showing that $K \leq_{s}$ $[k, \rightarrow)_{K}$.

Theorem 4.17. Scat has the $\Delta_{1}^{1}$-uniformization for epimorphisms.

Proof. We prove inductively on $\alpha$ that Scat ${ }_{\alpha}$ has the $\Delta_{1}^{1}$-uniformization for epimorphisms. Take $K \in \mathrm{Scat}_{0}$, so that there is $n \in \mathbb{N}$ such that $K=\{n\}$. For $m \in \mathbb{N}$ define $\tau_{n, m}: \mathbb{N} \rightarrow \mathbb{N} \cup\{*\}$ by letting $\tau_{n, m}(n)=m$, and $\tau_{n, m}(i)=*$ if $i \neq n$; then

$$
\begin{aligned}
\Phi_{K}: \text { Lin } & \longrightarrow \text { Epi } \\
L & \longmapsto \begin{cases}\tau_{n, m} & \text { if } L=\{m\} \text { for some } m \\
\perp & \text { otherwise }\end{cases}
\end{aligned}
$$

is a $\Delta_{1}^{1}$-uniformization a $K$.

Fix now $\alpha<\omega_{1}$ with $\alpha \geq 1$, and suppose that Scat $_{<\alpha}=\bigcup_{\beta<\alpha}$ Scat $_{\beta}$ has the $\Delta_{1}^{1}$-uniformization for epimorphisms. We need to prove that Scat $\operatorname{Sos}_{\alpha}$ has it too.

By Proposition 4.13 FinSum $\left(\right.$ Scat $\left._{<\alpha}\right)$ has the $\Delta_{1}^{1}$-uniformization for epimorphisms. Recall also that, by Fact 4.12 there is a $\Delta_{1}^{1}$ map that for any $L \in$ FinSum $\left(\operatorname{Scat}_{<\alpha}\right)$ chooses the code $\alpha_{L}$ of a $\Delta_{1}^{1}$-uniformization $\Phi_{L}$ for $L$.

Calling A the set of stable elements of Scat ${ }_{\alpha}$, Lemma 4.15yields that Scat ${ }_{\alpha}$ can be defined as

$$
\left.\left.\operatorname{FinSum}\left(\text { Scat }_{<\alpha}\right) \cup\left(\text { FinSum }_{\left(S_{c a t}\right.}\right)+A\right) \cup \star\left(\text { FinSum }_{<\alpha} \text { Scat }_{<\alpha}\right)+A\right),
$$

where we are using obvious notations.

Fact 4.11 and Proposition 4.13 tell us that if $\mathrm{A}$ has the $\Delta_{1}^{1}$-uniformization for epimorphisms, then so does Scat $\alpha$.

Fix $K \in \mathrm{A}$ and let $k_{0}$ be the minimum of $K$. If $K$ has a maximum, it is a singleton and we already know that it has a $\Delta_{1}^{1}$-uniformization. Thus we can assume that $K$ is a stable $\omega$-sum of elements of Scat ${ }_{<\alpha}$. To define a $\Delta_{1}^{1}(K)$-uniformization $\Phi_{K}$ of $K$ we use the $\Delta_{1}^{1}$-uniformizations $\Phi_{\left[k, k^{\prime}\right]_{K}}$ of $\left[k, k^{\prime}\right]_{K}$. Notice that the $\Phi_{\left[k, k^{\prime}\right]_{K}}$ are $\Delta_{1}^{1}(K)$ as well, because intervals are $\Delta_{1}^{0}(K)$.

We now define $\Phi_{K}(L)$ distinguishing three cases, each of them defined by a $\Delta_{1}^{1}$ property.

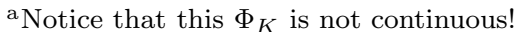


If $L$ has no minimum (a $\Pi_{2}^{0}$ condition) then notice that $L \not_{s} K$ and let $\Phi_{K}(L)=$ $\perp$.

If $L$ has two extrema (a $\Sigma_{2}^{0}$ condition) then notice that $L \leq_{s} K$ if and only if there is $k_{1} \in K$ such that $L \leq_{s}\left[k_{0}, k_{1}\right]_{K}$ holds, if and only if $\exists k_{1} \in K \Phi_{\left[k_{0}, k_{1}\right]_{K}}(L) \neq \perp$. In case $k_{1}$ does exist, choose $k_{1}$ minimal (as a natural number) and define $\Phi_{K}(L)$ by pieces from $\Phi_{\left[k_{0}, k_{1}\right]_{K}}(L)$ using the function DefPieces of Fact 4.6. Otherwise let $\Phi_{K}(L)=\perp$.

The last case is when $L$ has minimum but no maximum (a $\Delta_{3}^{0}$ condition). Let $\mathrm{B}=\{L \in \operatorname{Lin} \mid L$ has minimum but no maximum $\}$. If $L \in \mathrm{B}$ we denote by $\left\{l_{i} \mid i \in\right.$ $\mathbb{N}\}$ the canonical cofinite sequence in $L$, defined by letting $l_{0}$ be the minimum of $L$, and $l_{i+1}$ be the least (as natural number) $l \in L$ such that $l_{i}<_{L} l$. Notice that the map $L \mapsto\left\{l_{i} \mid i \in \mathbb{N}\right\}$ is $\Delta_{1}^{1}$ on the $\Delta_{3}^{0}$ set B.

Claim 4.18. There exists a $\Delta_{1}^{1}(K)$ function $f: \mathrm{B} \rightarrow K^{\mathbb{N}}$ such that, writing $f(L)(i)=k_{i}^{L}$, we have that the sequence $\left\{k_{i}^{L} \mid i \in \mathbb{N}\right\}$ is strictly increasing and cofinal in $K$ and moreover $L \leq_{s} K$ if and only if $\left[l_{i}, l_{i+1}\right]_{L} \leq_{s}\left[k_{i}^{L}, k_{i+1}^{L}\right]_{K}$ for every $i \in \mathbb{N}$.

Proof. Given $L \in \mathrm{B}$ we uniformly define in a $\Delta_{1}^{1}(K)$ way the sequence $\left\{k_{i}^{L} \mid i \in \mathbb{N}\right\}$ and an auxiliary sequence $\left\{m_{i}^{L} \mid i \in \mathbb{N}\right\} \in 2^{\mathbb{N}}$ by induction on $i$. The intuition for $m_{i}^{L}$ is that as long as $m_{i}^{L}=0$ we are still hoping to show that $L \leq_{s} K$, while when we set $m_{i}^{L}=1$ we actually know that $L \mathbb{L}_{s} K$ and we just need to make sure that $\left\{k_{i}^{L} \mid i \in \mathbb{N}\right\}$ is cofinal in $K$.

As $K \in \mathrm{B},\left\{k_{i} \mid i \in \mathbb{N}\right\}$ stands for the canonical cofinite sequence in $K$. First, $k_{0}^{L}=k_{0}$ is the minimum of $K$ and $m_{0}^{L}=0$. Assuming we have already defined $k_{i}^{L}$ and $m_{i}^{L}$ we proceed as follows. If $m_{i}^{L}=0$ we look for $k \in K$ such that $\left[l_{i}, l_{i+1}\right]_{L} \leq_{s}$ $\left[k_{0}^{L}, k\right]_{K}$. If we succeed, we let $k$ be the least (as natural number) such $k$ and, using the stability of $K$ and Fact 4.16, find the least (code for) a pair $\left(\hat{k}, k_{i+1}^{L}\right) \in K^{2}$ such that $\left[k_{0}^{L}, k\right]_{K} \leq_{s}\left[\hat{k}, k_{i+1}^{L}\right]_{K}$ and $\max _{K}\left(k_{i}^{L}, k_{i}\right) \leq_{K} \hat{k}$. This way we defined $k_{i+1}^{L}$, and we set also $m_{i+1}^{L}=0$. Notice that in this case we have

$$
\left[l_{i}, l_{i+1}\right]_{L} \leq_{s}\left[k_{0}^{L}, k\right]_{K} \leq_{s}\left[\hat{k}, k_{i+1}^{L}\right]_{K} \leq_{s}\left[k_{i}^{L}, k_{i+1}^{L}\right]_{K} .
$$

If either the search for $k \in K$ such that $\left[l_{i}, l_{i+1}\right]_{L} \leq_{s}\left[k_{0}^{L}, k\right]_{K}$ fails or $m_{i}^{L}=1$, we let $m_{i+1}^{L}=1$ and $k_{i+1}^{L}$ be the least (as natural number) $k \in K$ such that $\max _{K}\left(k_{i}^{L}, k_{i}\right)<_{K} k$.

Since we made sure that $k_{i} \leq_{K} k_{i}^{L}$ the sequence $\left\{k_{i}^{L} \mid i \in \mathbb{N}\right\}$ is indeed cofinal in $K$.

Now notice that if $m_{i}^{L}=0$ for every $i$ then $\left[l_{i}, l_{i+1}\right]_{L} \leq_{s}\left[k_{i}^{L}, k_{i+1}^{L}\right]_{K}$ for every $i$; using a definition by pieces we find a witness to $L \leq_{s} K$. If instead $m_{i}^{L}=1$ for some $i$ let $i$ be the least such. Then $\left[l_{i}, l_{i+1}\right]_{L} \mathbb{L}_{s}\left[k_{i}^{L}, k_{i+1}^{L}\right]_{K}$ and there is no $k \in K$ such that $\left[l_{i}, l_{i+1}\right]_{L} \leq_{s}\left[k_{0}^{L}, k\right]_{K}$. The latter fact implies $L \not \leq_{s} K$.

Now, using the claim, we can define $\Phi_{K}$ on B. If $\left[l_{i}, l_{i+1}\right]_{L} \leq_{s}\left[k_{i}^{L}, k_{i+1}^{L}\right]_{K}$ for every $i$ (a $\Delta_{1}^{1}(K)$ condition) then $\Phi_{K}(L)$ can be defined applying the function 
DefPieces from Fact 4.6 to the epimorphisms $\Phi_{\left[k_{i}^{L}, k_{i+1}^{L}\right]_{K}}\left(\left[l_{i}, l_{i+1}\right]_{L}\right)$. If instead $\left[l_{i}, l_{i+1}\right]_{L} \mathbb{S}_{s}\left[k_{i}^{L}, k_{i+1}^{L}\right]_{K}$ for some $i$ we set $\Phi_{K}(L)=\perp$.

We can finally pinpoint the complexity of StS, but first the complexity of StS $\cap$ Scat.

Corollary 4.19. The set of scattered strongly surjective orders is $\Pi_{1}^{1}$ and $\Pi_{1}^{1}$ complete.

Proof. The fact that StS $\cap$ Scat is $\boldsymbol{\Pi}_{1}^{1}$-hard is contained in Proposition 3.2 .

Given an order $K$ say that a $\Delta_{1}^{1}$-uniformization $\Phi$ of $K$ is strong if for all $L$ such that $L \leq_{i} K$ we have $\Phi(L) \neq \perp$. By Theorem $4.17 K$ is scattered and strongly surjective if and only if it is scattered and admits a strong $\Delta_{1}^{1}$-uniformization.

This gives in turn, using Fact 4.1, a $\Pi_{1}^{1}$ definition of StS $\cap$ Scat.

Corollary 4.20. The set $\mathrm{StS}$ is the union of a $\Sigma_{1}^{1}$ set and $a \Pi_{1}^{1}$ set. It is in particular $\check{\mathrm{D}}_{2}\left(\boldsymbol{\Pi}_{1}^{1}\right)$, and in fact $\check{\mathrm{D}}_{2}\left(\boldsymbol{\Pi}_{1}^{1}\right)$-complete.

Proof. By Proposition 3.3 Corollary 4.19 and Theorem 3.11

\section{Looking for uncountable strongly surjective orders}

\subsection{Classical examples are not strongly surjective}

Recall that Proposition 2.5(4) states that a strongly surjective linear order can have at most the cardinality of the continuum. Here we show that the most common orders of size the continuum and those that can be obtained from them using basic operations are not strongly surjective. We use different techniques, and for some linear orders we have different proofs that they are not strongly surjective.

We first give a cardinality obstruction for strong surjectivity of suborders of $\mathbb{R}$.

Theorem 5.1. Let $\aleph_{0}<\kappa \leq 2^{\aleph_{0}}$, and assume $2^{\aleph_{0}}<2^{\kappa}$. Then no $X \subseteq \mathbb{R}$ of cardinality $\kappa$ can be strongly surjective.

Proof. We use a counting argument: there are more subsets of $X$ than orderpreserving maps from $X$ to $X$.

Since $X \subseteq \mathbb{R}$ we can find a countable subset $D$ of $X$ such that for all $x, x^{\prime} \in X$, if $x<x^{\prime}$ holds then there is a $d \in D$ with $x \leq d \leq x^{\prime}$, and such that moreover the endpoints of $X$ belong to $D$, if they exist. Every order-preserving map from $X$ to $X$ is the extension of an order-preserving map from $D$ to $X$, and there are at most continuum many of those:

$$
\mid\{f: D \rightarrow X \mid f \text { order-preserving }\}|\leq| X^{D} \mid=\kappa^{\aleph_{0}}=2^{\aleph_{0}} .
$$

Fix now $f: D \rightarrow X$ order-preserving, and compute how many order-preserving extensions $g: X \rightarrow X$ of $f$ there can be. Take $x \in X \backslash D$, we can pick for $g(x)$ any 
$y \in X$ that satisfies

$$
\forall d \in(\leftarrow, x)_{X} \cap D \forall d^{\prime} \in(x, \rightarrow)_{X} \cap D f(d) \leq y \leq f\left(d^{\prime}\right) .
$$

Call $I_{x}$ the convex set of all points $y$ satisfying (5.1). If $I_{x}$ is trivial, that is empty or reduced to a singleton, then there is at most one order-preserving extension of $f$ to $x$. Notice now that by the properties of $D$, for $x \neq x^{\prime}$ in $X \backslash D$ the sets $I_{x}$ and $I_{x^{\prime}}$ are disjoint. Since $X \subseteq \mathbb{R}$ there can be only countably many non-trivial $I_{x}$, and each of these yields at most $\kappa$ possible extensions, so for a fixed $f$ we have

$$
\mid\{g: X \rightarrow X \mid g \text { order-preserving and } f \subset g\} \mid \leq \kappa^{\aleph_{0}}=2^{\aleph_{0}} .
$$

All in all we have at most continuum many order-preserving maps from $X$ to $X$, but $X$ has even more subsets by hypothesis so it cannot be strongly surjective.

Corollary 5.2. $\mathbb{R}$ and $\mathbb{R} \backslash \mathbb{Q}$ are not strongly surjective.

Theorem 5.1 and its proof do not provide a concrete $L$ such that $L \leq_{i} \mathbb{R}$ yet $L \leq_{s} \mathbb{R}$ (and similarly for $\mathbb{R} \backslash \mathbb{Q}$ ). A useful technique to prove that a linear order does not admit epimorphisms onto another one is to compare their gaps. Recall that a gap of $K$ is given by a non-empty initial segment $A \subseteq K$ with no maximum such that $K \backslash A$ is non-empty and has no minimum. Let $G(K)$ be the set of gaps of $K$ linearly ordered by $\subseteq$.

Proposition 5.3. If $L$ and $K$ are linear orders such that $L \leq_{s} K$ then $G(L) \leq_{i}$ $G(K)$.

Proof. If $f$ is an epimorphism from $K$ onto $L$ then $A \mapsto f^{-1}(A)$ is an injection from $G(L)$ to $G(K)$.

Corollary 5.4. Any linear order $L$ with $|G(L)|<2^{\aleph_{0}}$ is such that $\mathbb{Q} \not_{s} L$.

Hence $\mathbb{Q}$ witnesses the fact that $\mathbb{R}, \mathbb{R} \backslash \mathbb{Q}, \mathbb{Z}^{\mathbb{N}}$, and, for every countable $\alpha \geq \omega$, $2^{\alpha}$ ordered lexicographically are not strongly surjective.

Proof. The first part follows immediately from the Proposition because $|G(\mathbb{Q})|=$ $2^{\aleph_{0}}$.

Each of the linear orders considered in the second part of the statement (and indeed each short uncountable linear order) is non-scattered. Now observe that $\mathbb{R}$ and $2^{\alpha}$ are complete (that is, they have no gaps), while $|G(\mathbb{R} \backslash \mathbb{Q})|=\left|G\left(\mathbb{Z}^{\mathbb{N}}\right)\right|=\aleph_{0}$. The statements about $\mathbb{R}$ and $\mathbb{R} \backslash \mathbb{Q}$ are obvious.

To see that $2^{\alpha}$ is complete let $A$ be a non-empty subset of $2^{\alpha}$. Define inductively $x \in 2^{\alpha}$ as follows: given $\beta \in \alpha$ and assuming that $x(\gamma)$ has been constructed for every $\gamma \in \beta$, let $x(\beta) \in\{0,1\}$ be the least value such that $\left.x\right|_{\beta+1}$ majorizes $\left\{\left.z\right|_{\beta+1} \mid z \in A\right\}$. Then $x$ majorizes $A$. Moreover, if $y$ majorizes $A$ and $y<_{\text {lex }} x$, let $s=y \cap x$; then $s 0 \subseteq y, s 1 \subseteq x$, contrary to the definition of $x$. So $x=\sup A$.

To see that $\mathbb{Z}^{\mathbb{N}}$ has only countably many gaps let $L=\mathbb{Z}^{\mathbb{N}} \cup\left(\mathbb{Z}^{<\mathbb{N}} \backslash\{\emptyset\}\right)$ where the order on $\mathbb{Z}^{\mathbb{N}}$ is extended to $L$ by ordering $\mathbb{Z}^{<\mathbb{N}} \backslash\{\emptyset\}$ lexicographically and letting 
$x<_{L} s$ if and only if $\left.x\right|_{\ln (s)} \leq_{\text {lex }} s$ for every $x \in \mathbb{Z}^{\mathbb{N}}$ and $s \in \mathbb{Z}^{<\mathbb{N}} \backslash\{\emptyset\}$. Then $L$ is complete.

Notice that the fact that $2^{\mathbb{N}}$ and $\mathbb{Z}^{\mathbb{N}}$ are not strongly surjective shows that Corollary 2.14 stating that strongly surjective orders are closed under finite products, cannot be extended to infinite products.

The next natural candidates for being uncountable strongly surjective orders are the finite products obtained by using $\mathbb{R} \backslash \mathbb{Q}, \mathbb{R}$ and possibly some countable orders as factors. We show however that no uncountable strongly surjective order can be obtained in this way.

Lemma 5.5. Let $K, L$ and $M$ be linear orders. Suppose that $K \mathbb{L}_{s} L$ and that $K^{\prime} \mathbb{Z}_{s} M$ for any convex subset $K^{\prime}$ of $K$ that has more than one point. Then we have $K \mathbb{L}_{s} M L$.

Proof. Suppose we have $\varphi \in \operatorname{Epi}(K, M L)$ and consider $f: L \rightarrow \mathcal{P}(K)$ defined by $f(r)=\varphi(M \times\{r\})$. There is some $r \in L$ such that $f(r)$ is not a singleton, otherwise $f$ would induce an epimorphism from $L$ onto $K$. But then $f(r) \leq_{s} M$ and $f(r)$ is a convex subset of $K$ with more than one point, which is again impossible.

Definition 5.6. If $\kappa$ is an infinite cardinal, a linear order $L$ is $\kappa$-dense if it has no end points and between any two distinct elements of $L$ there are exactly $\kappa$ elements of $L$.

Lemma 5.7. There is a $2^{\aleph_{0}}$-dense suborder $M$ of $\mathbb{R} \backslash \mathbb{Q}$ such that every interval $[x, y]_{M}$, for $x<_{M} y$, has $2^{\aleph_{0}}$ gaps.

Proof. The following construction is a variation on the classical construction of a Bernstein set (see e.g. Example 8.24 in [8]).

Let $\left\{I_{n}\right\}_{n \in \mathbb{N}}$ be the set of the traces on $\mathbb{R} \backslash \mathbb{Q}$ of the real intervals with rational endpoints. In each $I_{n}$ we define subsets $X_{n}=\left\{x_{\beta}^{n}\right\}_{\beta \in 2^{\aleph_{0}}}$ and $Y_{\beta}^{n}=\left\{y_{\beta m}^{n}\right\}_{m \in \mathbb{N}}$, for $\beta \in 2^{\aleph_{0}}$, such that the elements $x_{\beta}^{n}, y_{\beta m}^{n}$ are all distinct. Fix $\beta<2^{\aleph_{0}}$ and $n \in \mathbb{N}$. Suppose that $x_{\beta^{\prime}}^{n^{\prime}}$ and $y_{\beta^{\prime} m}^{n^{\prime}}$ are defined for all $\beta^{\prime}<\beta$ and $n^{\prime}, m \in \mathbb{N}$, as well as $x_{\beta}^{n^{\prime}}$ and $y_{\beta m}^{n^{\prime}}$ for $n^{\prime}<n$ and $m \in \mathbb{N}$. Notice that the set $A$ of these elements, if non-empty, has cardinality $\max \left(\aleph_{0},|\beta|\right)<2^{\aleph_{0}}$. So pick any $x_{\beta}^{n} \in I_{n} \backslash A$ and distinct $y_{\beta m}^{n} \in I_{n} \backslash\left(A \cup\left\{x_{\beta}^{n}\right\}\right)$ such that

$$
\sup \left\{y_{\beta m}^{n} \mid m \in \mathbb{N}, y_{\beta m}^{n}<x_{\beta}^{n}\right\}=x_{\beta}^{n}=\inf \left\{y_{\beta m}^{n} \mid m \in \mathbb{N}, x_{\beta}^{n}<y_{\beta m}^{n}\right\} .
$$

It follows that every $x_{\beta}^{n}$ is a gap in $M=(\mathbb{R} \backslash \mathbb{Q}) \backslash\left\{x_{\beta}^{n} \mid \beta<2^{\aleph_{0}}, n \in \mathbb{N}\right\}$. Moreover, if $x, y \in M, x<y$, then $[x, y]_{M}$ contains some $I_{n} \cap M$, which has the cardinality of the continuum (containing all $y_{\beta m}^{n}$ ) and has continuum many gaps (at least every $\left.x_{\beta}^{n}\right)$. 
Theorem 5.8. Let $L=\prod_{0 \leq i \leq n} L_{i}$, where for each $i$ either $L_{i}$ is countable or $\mathbb{R} \backslash \mathbb{Q} \leq_{i} L_{i}$ and $\left|G\left(L_{i}\right)\right|<2^{\aleph_{0}}(\mathbb{R} \backslash \mathbb{Q}$ and $\mathbb{R}$ are instances of such linear orders). If $L$ is uncountable, then $L$ is not strongly surjective.

Proof. Suppose that $L$ is uncountable, so at least one of the factors is uncountable. Let $M$ be the order given by Lemma 5.7. Since $M \leq_{i} \mathbb{R} \backslash \mathbb{Q}$ we have $M \leq_{i} L$. It then suffices to show that $M \mathbb{L}_{s} L$, and this can be done by induction on $n$.

For $n=0$, notice that $M \not_{s} L$ since $|G(L)|<2^{\aleph_{0}}=|G(M)|$.

If the statement holds for $n$, let $L=\prod_{0 \leq i \leq n+1} L_{i}$. If $\prod_{1 \leq i \leq n+1} L_{i}$ is countable (by cardinality reasons), or by inductive hypothesis, $M \mathbb{Z}_{s} \prod_{1 \leq i \leq n+1} L_{i}$. Moreover, if $M^{\prime}$ is any convex subset of $M$ containing more than one point, then $M^{\prime} \mathbb{S}_{s} L_{0}$ either by cardinality reasons (if $L_{0}$ is countable) or by the fact that $M^{\prime}$ has more gaps than $L_{0}$ (if $L_{0}$ is uncountable). Now apply Lemma 5.5.

We already argued that $2^{\mathbb{N}}$ and $\mathbb{Z}^{\mathbb{N}}$ ordered lexicographically are not strongly surjective. However the gap method does not apply to other natural infinite lexicographic products, such as $\mathbb{Q}^{\mathbb{N}}, \mathbb{R}^{\mathbb{N}}$, and $(\mathbb{R} \backslash \mathbb{Q})^{\mathbb{N}}$, which have $2^{\aleph_{0}}$ many gaps. First we show that products such as $\mathbb{R}^{\mathbb{N}}$ and $(\mathbb{R} \backslash \mathbb{Q})^{\mathbb{N}}$ are not strongly surjective.

Theorem 5.9. For every $k \in \mathbb{N}$ let $L_{k}$ be a linear order with at least two elements such that for every convex set $K \subseteq L_{k}$ we have $\mathbb{Q} \mathbb{L}_{s} K$. Then $\mathbb{Q} \mathbb{Z}_{s} \prod_{k \in \mathbb{N}} L_{k}$, where the product is ordered lexicographically.

Proof. First of all notice that the hypothesis on $L_{k}$ implies that every convex subset of $L_{k}$ does not surject onto $1+\mathbb{Q}, \mathbb{Q}+1$, or $1+\mathbb{Q}+1$.

Suppose $f: \prod_{k \in \mathbb{N}} L_{k} \rightarrow \mathbb{Q}$ is an epimorphism. Our goal is to define an embedding $g: 2^{\mathbb{N}} \rightarrow \prod_{k \in \mathbb{N}} L_{k}$ such that $f$ is injective on the range of $g$, thus reaching a contradiction because $\mathbb{Q}$ is countable.

If $s \in \prod_{i<k} L_{i}$ for some $k \in \mathbb{N}$ we let $N_{s}=\left\{z \in \prod_{k \in \mathbb{N}} L_{k} \mid s \subset z\right\}$. To define $g$ we define $h: 2^{<\mathbb{N}} \rightarrow \bigcup_{k \in \mathbb{N}} \prod_{i<k} L_{i}$ such that $h(s) \in \prod_{i<2 k} L_{i}$ when $s \in 2^{k}$, $h(s) \subset h(t)$ when $s \subset t, f\left(N_{h(s)}\right)$ is a convex subset of $\mathbb{Q}$ with at least two elements, and $f\left(N_{h(s\urcorner 0)}\right) \cap f\left(N_{h(s\urcorner 1)}\right)=\emptyset$. Then we set $g(x)=\bigcup_{k \in \mathbb{N}} h(x\lceil k)$, so that it is immediate that if $x, y \in 2^{\mathbb{N}}$ are distinct then $f(g(x)) \neq f(g(y))$.

The definition of $h(s)$ is by recursion on the length of $s$, starting from $h(\emptyset)=\emptyset$. Thus we assume that $s$ has length $k$ and $h(s)$ is defined respecting the conditions, so that $f\left(N_{h(s)}\right)$ is isomorphic to one of $\mathbb{Q}, 1+\mathbb{Q}, \mathbb{Q}+1$, and $1+\mathbb{Q}+1$. Consider the map that sends $\ell \in L_{2 k}$ to $f\left(N_{h(s)-\ell}\right) \subseteq \mathbb{Q}$ : since $f\left(N_{h(s)}\right) \mathbb{L}_{s} L_{2 k}$, for some $\ell_{0} \in L_{2 k}$ we have that $f\left(N_{h(s)-\ell_{0}}\right)$ is not a singleton. Since $L_{2 k}$ has at least two elements $\ell_{0}$ is either not the maximum or not the minimum of $L_{2 k}$. Let us assume it is not the maximum (otherwise we reason symmetrically). Then $\left(\ell_{0}, \rightarrow\right)_{L_{2 k}}$ is a nonempty convex subset of $L_{2 k}$ and hence does not surject onto $\bigcup_{\ell>_{L_{2 k}} \ell_{0}} f\left(N_{h(s)-\ell) \text {, which }}\right.$ is isomorphic to one of $\mathbb{Q}, 1+\mathbb{Q}, \mathbb{Q}+1$, and $1+\mathbb{Q}+1$. Therefore we can find 
$\ell_{1}>_{L_{2 k}} \ell_{0}$ such that $f\left(N_{h(s)-\ell_{1}}\right)$ is also not a singleton. Notice that it might be that $f\left(N_{h(s)-\ell_{1}}\right)$ intersect $f\left(N_{h(s)-\ell_{0}}\right)$ in a common endpoint. Thus we go to the next level and find $\ell_{00}<_{L_{2 k+1}} \ell_{01}<_{L_{2 k+1}} \ell_{10}<_{L_{2 k+1}} \ell_{11}$ such that $f\left(N_{h(s)-\ell_{i} \ell_{i j}}\right)$ is not a singleton for every $i, j$. Consequently $f\left(N_{h(s)-\ell_{0} \ell_{00}}\right) \cap f\left(N_{h(s)-\ell_{1} \ell_{11}}\right)=\emptyset$. Let $h\left(s^{\wedge} i\right)=h(s)^{\frown} \ell_{i} \ell_{i i}$ for $i=0,1$.

Corollary 5.10. $\mathbb{Q}$ witnesses that $2^{\mathbb{N}}, \mathbb{Z}^{\mathbb{N}}, \mathbb{R}^{\mathbb{N}}$, and $(\mathbb{R} \backslash \mathbb{Q})^{\mathbb{N}}$ ordered lexicographically are not strongly surjective.

Proof. First notice that $2, \mathbb{Z}, \mathbb{R}$, and $\mathbb{R} \backslash \mathbb{Q}$ satisfy the condition imposed by Theorem 5.9 on the $L_{i}$ 's. Then observe that each of $2^{\mathbb{N}}, \mathbb{Z}^{\mathbb{N}}, \mathbb{R}^{\mathbb{N}}$, and $(\mathbb{R} \backslash \mathbb{Q})^{\mathbb{N}}$ is nonscattered.

To show that $\mathbb{Q}^{\mathbb{N}}$ is not strongly surjective we must use a different approach (obviously $\mathbb{Q} \leq_{s} \mathbb{Q}^{\mathbb{N}}$ ): we exploit the definability of epimorphisms in certain settings.

Theorem 5.11. No uncountable Borel suborder of $2^{\mathbb{N}}$, with the lexicographic order, is strongly surjective.

Proof. First, notice that the usual product topology and the order topology on $2^{\mathbb{N}}$ coincide. Indeed, for any $s \in 2^{<\mathbb{N}}$, the basic open set $N_{s}=\left\{x \in 2^{\mathbb{N}} \mid s \subseteq x\right\}$ is open in the order topology:

- $N_{\emptyset}=2^{\mathbb{N}}$

- if $s=s^{\prime} 01^{h}$ for some $h>0$, then $N_{s}=\left(s^{\prime} 01^{h-1} 01^{\infty}, s^{\prime} 10^{\infty}\right)_{2^{\mathrm{N}}}$; similarly if $s=s^{\prime} 10^{h}$ for some $h>0$

- if $s=0^{h}$ for some $h>0$, then $N_{s}=\left(\leftarrow, 0^{h-1} 10^{\infty}\right)_{2^{\mathbb{N}}}$; similarly if $s=1^{h}$ for some $h>0$

Conversely, given any $x \in 2^{\mathbb{N}}$, fix $y \in(x, \rightarrow)_{2^{\mathbb{N}}}$ and set $s=x \cap y$. Observe that $x \in N_{s 0}$ and $y \in N_{s 1}$, so $N_{s 1} \subseteq(x, \rightarrow)_{2^{\mathbb{N}}}$, which implies that $(x, \rightarrow)_{2^{\mathbb{N}}}$ is open in Cantor space. Similarly one proves that $(\leftarrow, x)_{2^{\mathbb{N}}}$ is open.

Now let $X \subseteq 2^{\mathbb{N}}$, and fix any order-preserving function $f: X \rightarrow 2^{\mathbb{N}}$. For any $s \in 2^{<\mathbb{N}}$ the subset $f^{-1}\left(N_{s}\right)$ of $X$ is convex, so there exists a convex subset $A$ of $2^{\mathbb{N}}$ such that $f^{-1}\left(N_{s}\right)=A \cap X$. By the above, if $x \in A$ and $x$ is not an end point of $A$, then $x$ is in the topological interior of $A$. This implies that $A$ is the union of an open set plus at most two points (its end points, if they exist), so $A$ is Borel in $2^{\mathbb{N}}$ and $f^{-1}\left(N_{s}\right)$ is Borel in $X$; consequently, $f$ is Borel.

If $X$ is Borel in $2^{\mathbb{N}}$, then $f(X)$ must be analytic; so if $X$ is uncountable, then $X$ is not strongly surjective, since there exist non-analytic subsets of $X$ onto which there can be no epimorphism.

Corollary 5.12. $2^{\mathbb{N}}, \mathbb{Z}^{\mathbb{N}}$ and $\mathbb{Q}^{\mathbb{N}}$ ordered lexicographically are not strongly surjective. 
Proof. It is enough to show that $\mathbb{Z}^{\mathbb{N}}$ and $\mathbb{Q}^{\mathbb{N}}$, endowed with the product topology on the discrete topology, Borel embed order preservingly in $2^{\mathbb{N}}$ ordered lexicographically. Since the natural inclusion is a Borel order preserving embedding of $\mathbb{Z}^{\mathbb{N}}$ into $\mathbb{Q}^{\mathbb{N}}$, by the main theorem of $\left[10\right.$ it is enough to prove that $2^{\omega+1} \mathbb{Z}_{i} \mathbb{Q}^{\mathbb{N}}$. Notice that $2^{\omega+1}$ has uncountably many pairs of consecutive points, so if $2^{\omega+1} \leq_{i} \mathbb{Q}^{\mathbb{N}}$ then $\mathbb{Q}^{\mathbb{N}}$, being dense, should have uncountably many pairwise disjoint open intervals. However this is not the case, as every $\leq_{l e x}$-open interval in $\mathbb{Q}^{\mathbb{N}}$ contains a non-empty open subset in the Polish topology of $\mathbb{Q}^{\mathbb{N}}$.

The argument of Theorem 5.11 cannot be extended to suborders of $2^{\alpha}$ for $\omega+1 \leq$ $\alpha<\omega_{1}$, since on $2^{\alpha}$ the order topology and the product topology do not coincide. In fact, for $\omega+1 \leq \alpha<\omega_{1}$, there are more order-preserving functions from $2^{\alpha}$ into itself than Borel maps with respect to the product topology. To see this, for every $A \subseteq 2^{\mathbb{N}}$ consider the function $\varphi_{A}: 2^{\alpha} \rightarrow 2^{\omega+1}$ defined by letting

$$
\varphi_{A}(x)= \begin{cases}\left(x\lceil\omega)^{-} 0\right. & \text { if } x\lceil\omega \in A ; \\ \left(x\lceil\omega)^{\wedge} 1\right. & \text { if } x\lceil\omega \notin A .\end{cases}
$$

The function $\varphi_{A}$ is order-preserving, and $\varphi_{A} \neq \varphi_{B}$ whenever $A \neq B$. Since $2^{\omega+1}$ is isomorphic to a suborder of $2^{\alpha}$, this shows that there are at least $2^{2^{\aleph_{0}}}$ orderpreserving functions from $2^{\alpha}$ into itself.

\subsection{Beyond $Z F C$}

In contrast with the negative results that we showed so far, we now build an uncountable strongly surjective order under extra set theoretic assumptions. For $\kappa$ an infinite cardinal less than the continuum, consider the statement

$\mathrm{BA}_{\kappa}$ : up to isomorphism, there is a unique $\kappa$-dense suborder of $\mathbb{R}$.

We know that $\mathrm{BA}_{\aleph_{0}}$ holds in ZFC, while the consistency of $\mathrm{BA}_{\aleph_{1}}$ with ZFC was proved in [2]. Moreover, $\mathrm{BA}_{\aleph_{1}}$ follows from PFA. The interest for $\mathrm{BA}_{\aleph_{2}}$ was rekindled recently, as witnessed by [12. Itay Neeman recently announced a proof of the consistency of $\mathrm{BA}_{\aleph_{2}}$ from large cardinals.

Theorem 5.13. Let $\kappa$ be an uncountable cardinal smaller than the continuum. Assume $\mathrm{BA}_{\kappa}$. Then there exist strongly surjective orders of cardinality $\kappa$.

We prove in fact the following.

Proposition 5.14. Let $\kappa$ be an uncountable cardinal smaller than the continuum. Suppose that, up to isomorphism, $X$ is the unique $\kappa$-dense suborder of $\mathbb{R}$. Then $L \leq_{s} X$ for any $L \subseteq \mathbb{R}$ with $|L| \leq \kappa$.

Proof. Let $M \subseteq L$ be a countable dense set in the order topology of $L$ containing all points having an immediate successor or an immediate predecessor in $L$ and the 
endpoints of $L$ if they exist. Let $Y=\sum_{l \in L} Y_{l}$, where $Y_{l}$ is isomorphic to $X$ if $l \in M$, and a singleton otherwise. It is then enough to show that $Y$ is isomorphic to $X$. It is easily checked that $Y$ is $\kappa$-dense, so it remains to check that $Y \leq_{i} \mathbb{R}$.

For each $l \in M$ let $Z_{l} \simeq \mathbb{R}$ be a linear order containing $Y_{l}$. If $l \in L \backslash M$, let $Z_{l}=Y_{l}$. Finally, let $Z$ be the completion of $\sum_{l \in L} Z_{l}$. The proof will be concluded by showing that $Z \simeq \mathbb{R}$.

By construction, $Z$ does not have minimum nor maximum and it is complete. To apply the classical characterization of the order type of $\mathbb{R}$ ([15], Theorem 2.30) it remains to show that $Z$ is separable. For each $l \in M$, pick a countable dense subset $Q_{l}$ of $Z_{l}$. Then $\sum_{l \in M} Q_{l}$ is dense in $Z$.

Applying Corollary 2.14 one obtains that if $X$ is the order provided by $\mathrm{BA}_{\kappa}$, then each $X^{n}$ is a strongly surjective order. These are in fact distinct order types, more precisely the following holds.

Proposition 5.15. Assume $\mathrm{BA}_{\kappa}$ and let $X$ witness it. Then

$$
X<_{i} X^{2}<_{i} X^{3}<_{i} \ldots \text { and } X<_{s} X^{2}<_{s} X^{3}<_{s} \ldots
$$

Proof. Since $X^{n} \leq_{s} X^{n+1}$, it is enough to show inductively $X^{n+1} \mathbb{Z}_{i} X^{n}$. First notice that $X^{2} \not_{i} X$, because $X^{2}$ contains an uncountable family of pairwise disjoint open intervals, while $X$ does not.

Now assume $X^{n+1} \mathbb{Z}_{i} X^{n}$, and suppose towards a contradiction that $f: X^{n} \times$ $X^{2} \rightarrow X^{n} \times X$ witnesses $X^{n+2} \leq_{i} X^{n+1}$. Write $f(a, x)=(g(a, x), h(a, x))$. If for some $a \in X^{n}$ the map $x \mapsto h(a, x)$ is injective then it witnesses $X^{2} \leq_{i} X$, contradicting the base step of our induction. Hence for all $a \in X^{n}$ there exist distinct $x, y \in X^{2}$ such that $h(a, x)=h(a, y)$. Fix $a \in X^{n}$, pick $x=\left(x_{0}, x_{1}\right)$ and $y=\left(y_{0}, y_{1}\right)$ with this property, and such that $x<_{X^{2}} y$. For all $z \in(x, y)_{X^{2}}$ and $b \in X^{n}$ we have $(a, x)<_{X^{n+2}}(b, z)<_{X^{n+2}}(a, y)$ and hence $h(b, z)=h(a, x)$. This implies that $(b, z) \mapsto g(b, z)$ is injective and witnesses $X^{n} \times(x, y)_{X^{2}} \leq_{i} X^{n}$. Now notice that $X \leq_{i}(x, y)_{X^{2}}$ : this is clear if $x_{1}<_{X} y_{1}$, while when $x_{1}=y_{1}$ it follows from the fact that $\left(x_{0}, y_{0}\right)_{X}$ is $\kappa$-dense, and hence isomorphic to $X$. Putting all together, we have shown that $X^{n+1} \leq_{i} X^{n}$, against the induction hypothesis.

In Theorem 5.13 we proved the existence of uncountable strongly surjective orders under a consequence of PFA. So it is natural to try to build strongly surjective orders under quite orthogonal principles, like $\diamond$ and its variations. To this end it appears that the following notions, with roots in [3], are relevant.

Definition 5.16. A partial order $\left(T, \leq_{T}\right)$ (often denoted only by $T$ ) is a tree if for all $t \in T$ the initial interval $\left(\leftarrow, t{ }_{T}\right.$ has order-type an ordinal $\alpha$ called the length of $t$. The set of nodes of length $\alpha$ is the $\alpha$ th level of $T$ : we denote it by $T^{\alpha}$. The height of $T$ is the smallest ordinal $\alpha$ such that $T^{\alpha}$ is empty. Moreover we say that $s, t \in T$ belong to the same brotherhood of $T$ if $\left(\leftarrow, t\left[_{T}=(\leftarrow, s[T\right.\right.$. 
If every brotherhood of $T$ is linearly ordered, then we can order $T$ by the lexicographic order we denote by $\leq_{l e x}$ and, following Baumgartner [3], we say that $T$ is doubly ordered.

Two doubly ordered trees $T$ and $S$ are isomorphic (and we write $\left(T, \leq_{T}, \leq_{l e x}\right) \cong$ $\left.\left(S, \leq_{S}, \leq_{l e x}\right)\right)$ if there exists a bijection $f: T \rightarrow S$ which preserves both the partial and the linear orders.

Definition 5.17. A doubly ordered tree $\left(T, \leq_{T}, \leq_{l e x}\right)$ is a Baumgartner tree if the following conditions hold:

- $\left(T, \leq_{T}\right)$ is a Suslin tree (that is, every chain and every antichain in $T$ is countable, the height of $T$ is $\omega_{1}$, and for all $t \in T^{\alpha}$ and all $\beta<\omega_{1}$ with $\alpha \leq \beta$ there exists $s \in T^{\beta}$ such that $t \leq_{T} s$ ).

- $T$ has rational brotherhoods, that is the ordering of each brotherhood of $T$ is isomorphic to $\mathbb{Q}$.

- for every $X \in[T]^{\aleph_{1}}$, if

- $X$ is cofinal with respect to $\leq_{T}$ in $\left\{t \in T \mid \exists s \in X\left(s \leq_{T} t\right)\right\}$,

- $X$ has a rational basis, that is the ordering of its minimal elements is isomorphic to $\mathbb{Q}$,

then we have $\left(X, \leq_{T}, \leq_{l e x}\right) \cong\left(T, \leq_{T}, \leq_{l e x}\right)$.

Assuming $\diamond^{+}$, in Theorem 4.15 of [3], Baumgartner claimed to build a minimal Specker type which was in fact the linear part of what we call a Baumgartner tree. As pointed out by Hossein Lamei Ramandi, Baumgartner's proof has however a gap: there are indeed many counterexamples to the crucial Lemma 4.14, stated without proof.

Recently, Dániel Soukup ([16, see Section 4) modified the proof of Theorem 2.3 in [7] to construct a Baumgartner tree under $\diamond^{+}$.

Theorem 5.18. The linear order of a Baumgartner tree is strongly surjective.

Proof. Fix a Baumgartner tree $\left(T, \leq_{T}, \leq_{l e x}\right)$ and let $L=\left(T, \leq_{l e x}\right)$.

Observe first that, as $T$ has rational brotherhoods, $\left(T^{0}, \leq_{l e x}\right) \simeq \mathbb{Q}$ holds. Hence $L$ can be written as a $\mathbb{Q}$-sum and $\mathbb{Q} \leq_{s} L$. For any countable linear order $K$ we have $K \leq_{s} \mathbb{Q}$ and hence $K \leq_{s} L$.

It remains to deal with the uncountable suborders of $L$. Given $X \subseteq T$ uncountable let $K=\left(X, \leq_{l e x}\right)$. Consider

$$
X^{\circ}=\left\{s \in X \mid X \text { is cofinal in }\left\{t \in T \mid s \leq_{T} t\right\}\right\},
$$

and call $A$ the set of its $\leq_{T}$-minimal elements. As $A$ is an antichain and $T$ is Suslin, $A$ is countable. Let $B=X \backslash X^{\circ}: B$ is not cofinal in any set of the form $\left\{t \in T \mid s \leq_{T} t\right\}$ and hence, by a well-known property of Suslin trees, it is countable. For $s \in B$ let $L_{s}$ be the singleton order, and for $s \in A$ let $L_{s}=1+L$. Notice that for $s \in A$ we have $L_{s} \simeq\left(\left\{t \in X \mid s \leq_{T} t\right\}, \leq_{\text {lex }}\right)$ by the last clause in the definition of Baumgartner 
tree. The set $\left\{t \in X \mid s<_{T} t\right\}$ has indeed a rational basis $C$ : take $t, t^{\prime} \in C$ with $t<_{\text {lex }} t^{\prime}$ and call $s^{\prime}=t \cap t^{\prime} \geq_{T} s$. The second clause implies that there are immediate successors $s_{0}<_{\text {lex }} s_{1}<_{\text {lex }} s_{2}<_{\text {lex }} s_{3}<_{\text {lex }} s_{4}$ of $s^{\prime}$ such that $s_{1} \leq_{T} t$ and $s_{3} \leq_{T} t^{\prime}$. Cofinality gives us then $t_{i} \geq_{T} s_{i}$ in $C$ for $i=0,2,4$, so $C$ is densely ordered with no extremes. As an antichain in $T, C$ is countable.

Thus we have $K \simeq \sum_{s \in A \cup B} L_{s}$, where the sum is taken according to lexicographic order. Since the 0th level of $T$ is ordered as $\mathbb{Q}$ we have $L \simeq L \mathbb{Q}$, so for any countable linear order $M$ we have, as linear orders: $L M \simeq(L \mathbb{Q}) M \simeq L(\mathbb{Q} M) \simeq$ $L \mathbb{Q} \simeq L$. In particular $L_{s} \simeq 1+L \leq_{s} L 2 \simeq L$ for every $s \in A$, so we have $\sum_{s \in A \cup B} L_{s} \leq_{s} L M$, where $M=\left(A \cup B, \leq_{\text {lex }}\right)$. Since $M$ is countable, $L M \simeq L$. Altogether we showed $K \leq_{s} L$, as needed.

\subsection{Some directions for further research}

In this paper, we have given a fairly complete treatment of countable strongly surjective linear orders. On the other hand, various problems regarding uncountable strongly surjective linear orders have been left open. We discuss briefly here some lines for further research in this direction.

\subsubsection{The existence of uncountable strongly surjective orders}

In the first draft of this paper, we left open three problems concerning strongly surjective orders. The main one was: Does there exist an uncountable strongly surjective order in ZFC? With two related questions: Does there exist an uncountable strongly surjective order under $\mathrm{CH}$ ? Or under $\diamond$ ?

Upon learning about these problems, Dániel Soukup answered negatively the second question, so a fortiori the first one: see Section 5 of [16.

The existence of a strongly surjective order under $\diamond$ remains open. Dániel Soukup included a healthy list of open problems about uncountable strongly surjective orders in Section 6 of [16].

\subsubsection{Definably strongly surjective orders}

In Theorem 5.11 we proved that no uncountable Borel suborder of $2^{\mathbb{N}}$ can be strongly surjective by using definability reasons: such an order cannot surject onto a nonanalytic suborder, since epimorphisms are Borel. This suggests that there may be some Borel subsets of $2^{\mathbb{N}}$ for which this is the only obstruction to strong surjectivity, as they admit epimorphisms onto all their analytic suborders. Call such orders definably strongly surjective.

Corollaries 5.4 and 5.10 (but not Corollary 5.12 ) show that $2^{\mathbb{N}}$ and $\mathbb{Z}^{\mathbb{N}}$ ordered lexicographically are not definably strongly surjective.

Question 5.19. Do there exist definably strongly surjective orders that are not strongly surjective? In particular, is $\mathbb{Q}^{\mathbb{N}}$ definably strongly surjective? Can the con- 
cept of a definably strongly surjective order be extended beyond the Borel suborders of $2^{\mathbb{N}}$ ?

\section{Acknowledgments.}

An early version of this work, and notably a boldface form of Theorem 4.17 was presented to the "Groupe de travail en théorie descriptive des ensembles" at University Paris 6 . We would like to thank the whole group for their patience, and Alain Louveau for suggesting the lightface version of Theorem 4.17, and pointing out that it would yield Corollary 4.19 .

The research presented in this paper has been done while the first author was visiting the Department of information systems of the University of Lausanne. He

wishes to thank the Équipe de logique, and in particular its director prof. Jacques Duparc, for providing such a friendly environment.

The research of the second author was funded by a fellowship from the Istituto Nazionale d'Alta Matematica (INdAM) and in part by FWF Grant P28153.

The research of the third author was supported by PRIN 2012 Grant "Logica, Modelli e Insiemi".

The paper was completed while all three authors were attending the workshop "Current trends in Descriptive Set Theory" at the Erwin Schrödinger International Institute for Mathematics and Physics in Vienna. The authors wish to thank the ESI for its support and hospitality.

Finally, we thank the anonymous referee for numerous remarks and suggestions.

\section{References}

[1] A. Andretta, R. Camerlo, Analytic sets of reals and the density function in the Cantor space, European Journal of Mathematics, to appear.

[2] J.E. Baumgartner, All $\aleph_{1}$-dense sets of reals can be isomorphic, Fundamenta Mathematicae 79 (1973), 101-106.

[3] J.E. Baumgartner, Order types of real numbers and other uncountable orderings, in I. Rival (ed.), Ordered sets, 1982, 239-277.

[4] R. Camerlo, R. Carroy, A. Marcone, Epimorphisms between linear orders, Order 32 (2015), 387-400.

[5] R.Camerlo, A. Marcone, L. Motto Ros, Polish metric spaces with fixed distance set, arXiv:1809.06588.

[6] K. Fournier, Wadge hierarchy of differences of co-analytic sets, The Journal of Symbolic Logic 81 (2016), 201-215.

[7] A. Hajnal, Z. Nagy, L. Soukup, On the number of certain subgraphs of graphs without large cliques and independent subsets, in A. Baker, B. Bollobás and A. Hajnal (eds.), A tribute to Paul Erdös, 1990, 223-248.

[8] A.S. Kechris, Classical descriptive set theory, Springer-Verlag 1995.

[9] C. Landraitis, A combinatorial property of the homomorphism relation between countable order types, The Journal of Symbolic Logic 44 (1979), 403-411.

[10] A. Louveau, Two results on Borel orders, The Journal of Symbolic Logic 54 (1989), 865-874.

[11] A. Louveau, Effective descriptive set theory, unpublished notes. 
32 Riccardo Camerlo, Raphaël Carroy, Alberto Marcone

[12] J.T. Moore, S. Todorcevic, Baumgartner's isomorphism problem for $\aleph_{2}$-dense suborders of $\mathbb{R}$, Archive for Mathematical Logic 56 (2017), 1105-1114.

[13] A. Montalbán, Indecomposable linear orderings and hyperarithmetic analysis, Journal of Mathematical Logic 6 (2006), 89-120.

[14] Y.N. Moschovakis, Descriptive set theory, American Mathematical Society 2009.

[15] J.G. Rosenstein, Linear orderings, Academic Press 1982.

[16] D.T. Soukup, Uncountable strongly surjective linear orders, Order, 2018. 